\title{
Optimal sizing of energy communities with fair revenue sharing and exit clauses: value, role and business model of aggregators and users
}

\author{
Davide Fioriti \\ DESTEC, Universitá di Pisa \\ Largo Lucio Lazzarino, 56122 Pisa, Italy \\ Antonio Frangioni \\ Dipartimento di Informatica, Universitá di Pisa \\ Largo B. Pontecorvo 3, 56127 Pisa \\ Davide Poli \\ DESTEC, Universitá di Pisa \\ Largo Lucio Lazzarino, 56122 Pisa, Italy
}

May 4, 2021

LICENSE: Creative Commons: Attribution-Noncommercial - No Derivative Works 



\title{
Optimal sizing of energy communities with fair revenue sharing and exit clauses: value, role and business model of aggregators and users
}

\author{
Davide Fioriti \\ DESTEC, Universitá di Pisa \\ Largo Lucio Lazzarino, 56122 Pisa, Italy \\ Antonio Frangioni \\ Dipartimento di Informatica, Universitá di Pisa \\ Largo B. Pontecorvo 3, 56127 Pisa \\ Davide Poli \\ DESTEC, Universitá di Pisa \\ Largo Lucio Lazzarino, 56122 Pisa, Italy
}

May 4, 2021

\begin{abstract}
Energy communities (ECs) are essential policy tools to meet the Energy Transition goals, as they can promote renewable energy sources, demand side management, demand response and citizen participation in energy matters. However, to fully unleash their potential, their design and scheduling requires a coordinated technical operation that the community itself may be ill-equipped to manage, in particular in view of the mutual technical and legal constraints ensuing from a coordinated design. Aggregators and Energy Service COmpanies (ESCOs) can perform this support role, but only provided that their goals are aligned to those of the community, not to incur in the agency problem.

In this study, we propose a business model for aggregators of ECs, and its corresponding technical optimization problem, taking into account all crucial aspects: i) alleviating the risk of the agency problem, ii) fairly distributing the reward awarded to the EC, iii) estimating the fair payment for the aggregator services, and iv) defining appropriate exit clauses that rule what happens when a user leaves the EC. A detailed mathematical model is derived and discussed, employing several fair and theoretically-consistent reward distribution schemes, some of which are, to the best of our knowledge, proposed here for the first time. A case study is developed to quantify the value of the aggregator and compare the coordinated solution provided by the aggregator with non-coordinated configurations, numerically illustrating the impact of the reward distribution schemes.

The results show that, in the case study, the aggregator enables reducing costs by $16 \%$ with respect to a baseline solution, and enables reaching $52.5 \%$ renewable share and about $46 \%$ self/shared consumption, whereas these same numbers are only $28-35 \%$ for the non-coordinated case. Our results suggest that the aggregator fair retribution is around $16-24 \%$ the added benefit produced with respect to the noncoordinated solution, and that stable reward distribution schemes such as Shapley/Core or Nucleolus are
\end{abstract}


recommended. Moreover, the results highlight the unwanted effect that some non-cooperative ECs may have an added benefit without providing any positive effect to the power system.

Our work lays the foundations for future studies on business models of aggregators for ECs and provides a methodology and preliminary results that can help policy makers and developers in tailoring national-level policies and market-offerings.

Keywords:Mixed Integer Linear Programming (MILP), hybrid Renewable/Citizenship Energy Community system (REC/CEC), coalition game theory, cooperative game, Energy Service COmpany (ESCO), Peak power management

\section{Nomenclature}

\section{Indices and sets}

$i \in I \quad$ index and set of the microgrid users

$j \in J \subseteq I \cup A$ Any subset of users contained in I, eventually including the aggregator A, and its index

$t \in T$ index and set of the time-step of the simulation

$w \in W$ index and set of payment periods of the peak power component for users and microgrid (i.e. one every month)

$\hat{t}_{w} \in \hat{T}_{w} \subseteq T$ index and subset of the indices $t$ referring to a peak-period $w$

$y \in Y$ index and set of the years of the investment

$a \in A_{j}$ set of the assets of user $j$; multiple types of technologies are taken into account in the following categories: battery $(\mathrm{B})$, battery converter $(\mathrm{C})$, and renewable assets $(\mathrm{R})$.

$b / c / r \in A_{j}^{B / C / R} \subseteq A_{j}$ index and subset of assets of user $j$, depending on technology type.

$c(b), b(c)$ Converter technology $c$ corresponding to the battery technology $b$ and viceversa: a single converter is coupled to each battery technology.

\section{Variables}

$P_{j, t}^{U, M+/ U, M-}$ Energy injected (+) or absorbed (-) at each user POD and contributing to the total energy shared within the EC

$P_{j, t}^{U, P+/ U, P-}$ Energy injected (+) or absorbed (-) at each user POD without being shared within the EC

$x_{j}^{a, U} \quad$ Installed capacity for each technology $a$ by the user $j$

$P_{j, t}^{R, U} \quad$ Total non-dispatchable renewable production for technology $r$ in time step $t$ of user $j$

$P_{j, t}^{c+/ c-, U}$ Discharging $(+)$ and charging (-) power of the battery converter in time step $t$ of user $j$ and technology $c$

$P_{j, w}^{U, m a x}$ Maximum power usage at the user POD $j$ 
$E_{j, t}^{b, U} \quad$ State of charge of the battery of the user (U) $j$ at time step $t$ and battery technology $b$

$\sigma \quad$ Fraction of the economic surplus generated by the EC kept by the aggregator

$\hat{\theta} \quad$ Economic margin of current coalition subset to remain in the grand-coalition with respect to creating their own energy community.

\section{Expressions}

$P_{j, t}^{U, P O D+/-}$ Power exchanged at the POD of user $j$ in time step $t$

$E_{t}^{S H} \quad$ Energy shared among users in the EC in time step $t$

$N P V^{A}(J / I)$ Net Present Value of the aggregator for a given set of users

$N P V^{U A}(J / I)_{j}$ Net Present Value including the reward of a member of the energy community composed by a set of users

$N P V_{j}^{U}$ Net Present Value of a user in the non-cooperative formulation

$S W^{t o t / U, N A / N C / A N C / C O}(J / I)$ Total (tot) or Users (U) social welfare in the NA/NC/ANC/CO case

$R_{j, t}^{U, P} \quad$ Net contribution to the electricity bill with respect to the public grid by user $j$

$R C_{j, y}^{U}$ Replacement costs of the assets by user $j$

$R V_{j, y}^{U}$ Recovery value of the assets by user $j$ at the end of the project

$C_{j, w}^{U, P} \quad$ Costs of the peak power for the user $j$ in every peak period $w$

$C A P E X_{j, a}^{U}$ Investment costs of user $j$ and asset $a$

$C_{j, a}^{U, M} \quad$ Yearly maintenance costs of device $a$ owned by user $j$

$\bar{P}_{j, t}^{U+/ U-}$ Upper bound of the power supplied (+) or absorbed (-) by user $j$ in time step $t$

$E C R_{y}(J)$ Reward awarded to the energy community.

$v(J) \quad$ Shared benefit created by the energy community with members $J$.

$V_{i}^{\text {exit }} \quad$ Exit cost to be paid by user $i$ when leaving the community

\section{Parameters}

$d \quad$ Discount factor

$\Delta \quad$ Time resolution

$N_{j}^{Y, a} \quad$ Lifetime of the components of technology $a$

$\pi_{t}^{P+} \quad$ Price received when electricity is injected into the public grid.

$\pi_{t}^{P-, V / F}$ Variable $(\mathrm{V})$ and fixed $(\mathrm{F})$ costs paid when electricity is absorbed from the public grid 
$\pi_{t}^{R} \quad$ Reward benefit awarded to the energy community for every energy unit consumed within the community

$P_{j, t}^{L} \quad$ Load demand of user $j$ in time step $t$

$p_{j, t}^{r, U} \quad$ Specific renewable production for technology $r_{j}$ of user $j$ at time step $t$

$\beta_{j}^{b, \max }, \beta_{j}^{b, \min }$ Maximum and minimum state of charge of the battery

$\eta_{j}^{b} \quad$ Roundtrip efficiency of the battery technology $b$ of user $j$

$c_{j}^{a, U} \quad$ Investment cost for each unit of technology $a$ for a user $j$

$c_{j}^{a, U, M}$ Maintenance cost of each unit of technology $a$ for the user $j$

$c_{w}^{P P} \quad$ Peak power tariff related to the national electricity market in each payment period $w$ $m_{t}^{T}, m_{w}^{W}$ Multiplier weights of peak power and energy costs

$N P V_{j}^{U, N C}$ Annualized profits of each user $j$ without the aggregate in the NC configuration.

$\bar{x}_{j}^{a, U} \quad$ Upper bound of the maximum installed capacity for each technology $a$ by the user $j$

$\eta_{j}^{L} \quad$ Efficiency of the distribution system between the user and the junction box

\section{Acronyms}

EC Energy Community

ESCO Energy Service COmpany

POD Point Of Delivery

NA No-Assets case

NC Non-Cooperative case

ANC Aggregated Non-Cooperative

$\mathrm{CO}$ COoperative case

NPV Net Present Value

NPC Net Present Cost

SW Social Welfare

SC Social Cost

CAPEX CAPital EXpenditure

OPEX OPerational EXpenditure

Sh Shapley Value reward distribution scheme 
Nuc Nucleolus reward distribution scheme

ShC Shapley value/Core reward distribution scheme

ShNuc Shapley value/Nucleolus reward distribution scheme

VarC MinVariance/Core reward distribution scheme

VarNuc MinVariance/Nucleolus reward distribution scheme

\section{Introduction}

\subsection{Motivation}

The need of meeting an increasing share of energy demand using renewable sources, combined with the uncertain production of wind and solar power plants, is raising pressure on the stability of existing power systems. The creation of the so-called Energy Communities (ECs), i.e., local, organized and coordinated aggregates of consumers and prosumers [21], is widely recognised as a useful tool to alleviate this burden, attract private financing, meet the environmental targets, and ultimately addressing the Energy Transition challenge [27].

ECs are an hot topic with a huge technical and economical potential. While there are no less than 3500 ECs in the European Union alone [10], and others in US, Canada and New Zeland [7, 23, 21], yet, a surge in the numbers is expected, as most countries have not finalized the adoption of specific policies [21, 27]. Being among the world leaders in Energy Transition, the EU has approved the first directive (RED II) on Renewable Energy Communities (REC) in mid 2018, followed by the IEMD directive that broadens the concept to that of "Citizenship Energy Community" (CEC) also accounting for sharing of non-renewable energy sources [27]. Yet, the full adoption of both directives is demanded to national governments, and most countries, among which Italy [4], have only approved temporary regulations while working to estimate social responses and tailor the best framework for their population. While ECs can create benefits, they may also lead to mutual constraints among users, including energy dependence, privacy issues, organizational problems and economic counterparts, with repercussions on the network grid and financing schemes. These have to be properly accounted for, since the initial design of the regulation acts issued by national authorities [7].

The literature regarding the optimal sizing of district energy systems or aggregated microgrids is rich [19]. However, most studies focus on the technical problem of finding the optimal solution and overlook that the creation of an energy community, while providing environmental and efficiency benefits, may lead to concerns regarding the ownership of common assets, the risks of users leaving the community, the fair distribution of profits, and possible barriers for users to join. Therefore, it is timely to address the issue of optimal sizing of ECs also accounting for these non-technical concerns. In this study, we propose a sizing methodology based on a custom business model for ECs that is aimed at stimulating the cooperation of users and the optimal operation of the EC by the community manager, or aggregator, also including exit clauses to rule how users can leave the community. The proposed business model highlights the role of aggregators and it is well aligned to current EC regulation and objectives. 


\subsection{Literature analysis}

The recent US [6] and EU [27] policies supporting renewable energy communities provide frameworks for energy sharing, thus unleashing the full potential of community microgrids and aggregators $[13,51,54,53,26]$.

Traditionally, aggregators are private operators that act on the energy market on behalf of a set of consumers and prosumers [41] to provide benefits by bidding/hedging on the market to achieve a lower energy purchase price, properly operating generation and storage systems, and/or implementing demandside management or demand response policies [45, 25, 8]. Given this purpose, most studies have traditionally focused on techniques to optimally operate the aggregate $[20,3,17]$, usually focusing on economic indicators. Some studies have focused at maximizing the social welfare of the aggregate, but overlooking how profits shall be distributed among participants $[16,15]$. Others, instead, have proposed game-theoretic approaches, be them cooperative or non-cooperative, to identify the best profit allocation $[41,46,18,33,11,52]$.

In [41], a non-cooperative approach for coordinating multiple electricity aggregators is proposed to provide additional flexibility in a network. A similar approach is proposed in [52] but for power-heating systems. Given the focus on storage for providing flexibility, competition between storage aggregators have been simulated in [30] and optimal bidding strategies have been estimated. In these non-cooperative studies, the players are often aggregators whose business profit hinges on trading in the electricity market. In the case of ECs, players (i.e, users) are typically not interested in direct trading, and they would rather delegate this role. As a consequence, a cooperative approach is more appropriate, especially considering the social focus of EC policies expressed by EU [27].

In cooperative formulations, the users cooperate with the goal of achieving the cheapest solution. According to the cooperative game theory, the stability of a coalition depends on the cost/profit allocation that shall adhere to two main principles: rationality and efficiency $[46,18]$. Rationality guarantees that no subset of users benefit from leaving the community, while efficiency specifies that all benefits given by the aggregate shall be completely distributed among all components of the aggregate. All solutions that are efficient and rational belong to the "Core", which is a key concept in game theory [1,2]. The Core definition is embedded in the Nucleolus concept adopted in [18] for the coordination of multiple microgrids in a distribution system: rationality and efficiency properties are satisfied, but concerns about fairness arise because the approach mainly focuses on strengthening the stability of the coalition. Moreover, the Nash Bargaining theory has also been proposed for reactive power compensation in distribution networks [33], where profits are shared equally among the players. However, these principles lead to a wide set of different allocation of costs and rewards [12], which shall be fair according to each player's contribution [40, 9]. In [48], a fair-efficient approach is proposed to distribute profits according to a modified Shapley value, which is a reference-key measure in economics for profit allocation in a coalition [14] but may suffer from stability issues [22]. The Owen sharing method is proposed in [32] to distribute the profits generated by the aggregated bidding of a group of wind turbines that makes use of dual variables to distribute profits. The Owen method uses the dual solution to distribute profits among the players in a linear production game; while Owen solution belongs to the core [36], they may not achieve desirable properties such as these of Nucleolus or Shapley. In [2] a min-variance allocation mechanism is discussed to distribute profits among an energy community where prosumers could install PV assets only. This approach may reduce computational time while achieving stability. Fewer studies have instead focused on combining the optimal sizing and operations of networked microgrids with different cost-allocation rules, such as Nash Bargaining, Shapley Value or Myerson Value [46, 37, 47]. In the case of 
Nash Bargaining, however, profits tend to be equally distributed among the players, which is not a desirable characteristic in that it does not account for the specific benefits provided by each user, and therefore it does not properly stimulate users in the installation of assets. On the other hand, the Myerson Value approach applies to partially connected grids [31], which is not the case for typical ECs. Nucleolus and Core concepts lead to efficient and rational profit allocation, but concerns about fairness and uniqueness arise; conversely, the Shapley Value is widely recognized as a fair measure, but rationality is not guaranteed [50]. For this reason, in this study we start from Core, Nucleolus, Shapley value and Min-Variance as the main distribution schemes and we propose some novel profit-sharing allocation methods that try to inherit the best characteristics of each.

Furthermore, most of the studies from the literature only focus on aggregate of users without discussing a complete business models for ECs that addresses all relevant concerns, i.e., optimal management for the community, remunerating the fair economic benefit to the aggregator, and the long-term sustainability of the community. This work aims at providing a comprehensive treatment of all these aspects.

According to the EU directives (RED-II and IEMD) [27], ECs shall be no-profit entities that incentive the participation into energy matters and promote the use of renewable energy with environmental benefits [10]. Accordingly, most of the literature considers the community manager to be any member of the EC whose objective is the maximisation of the social welfare of the community itself $[24,50,29]$. However, most members would not have the skills for properly sizing and coordinating the system. Most likely, the functions of the community manager will be externalized outside the community to an ESCO or an aggregator. This, however, requires defining a reward scheme for the community manager to properly compensate its skills and labour while ensuring that its objective does not deviate from the goals of the EC users, thus avoiding the "agency problem" [44]. In this study we discuss this concept in details and propose a business model, and a corresponding mathematical formulation, for the optimal design and management of the community power system that aims at aligning the aggregator's goal with the community's objective.

In any EC the problem of the assets' ownership inevitably also arises, and there are mainly two alternatives depending on the organization: the components are bought or leased by the entire community [5] or by a specific user [29]. Both options lead to intertwined financial (CAPEX/OPEX) and/or physical (shared assets) constraints that may be a barrier to create an EC. The general gist of the EU policies [27] is that citizens and companies shall be free to choose their personal affairs, as failure to ensure this may reduce the participation and ultimately hinder the financial stability of the community. Therefore, the membership contracts between each user and the EC shall have specific clauses, which we refer to as "exit" in our development, to address these concerns; this can have implications in terms of optimal design. We believe that this matter shall be properly taken into account, while the literature [46,37, 47] tends to overlook this hurdle and mainly focuses on the optimal operations of the system [40, 9], and sometimes its optimal design [24, 50, 29].

\subsection{Contributions}

The main contributions of our work are as follows:

1. development of several new reward allocation mechanisms (Shapley-Core, Shapley-Nucleolus and MinVariance-Nucleolus) and comparison to existing methodologies (Shapley, Nucleolus, Min/Variance) to reach efficient, rational and fair distribution of the reward received by the EC;

2. proposal of the exit clauses that clearly state at which conditions a user can leave the community; 
3. proposal and discussion of a business model to alleviate the "agency problem" in the design and management of ECs;

4. extensive comparison of different fair game theoretic mechanism to allocate the collective profits;

5. evaluation of the fair benefit generated by the aggregator, considered as a player contrary to standard approaches;

6. development of a mathematical optimization model to properly size the EC considering all the abovementioned novelties.

\subsection{Organization}

Our work is structured as follows. Section 2 introduces the organization of ECs and Section 3 describes the proposed business model for both ECs and aggregators. Game-theoretic methodologies to allocate among the users and the aggregator the profits generated by the EC are discussed in Section 4, and then implemented in the mathematical modelling in Section 5. The case study is presented in Section 6 and results are detailed in Section 7. Finally, conclusions are drawn.

\section{The organization of Energy Community}

\subsection{Description}

According to the EU regulation [10], an Energy Community is a no-profit entity whose participants take collective actions with the goal of possibly engaging all energy customers in energy matters and promote social innovation. Therefore, in terms of organisation, the EU directives do not pose significant constraints except that the entity "energy community" shall be no-profit [10], and that in the case of renewable energy communities (REC), shared energy shall be renewable [27]. Yet, these limitations are loosened up in the IEMD directive, with the definition of Citizen Energy Communities (CEC). EU countries have only recently started some initiatives issuing temporary regulations, e.g., in Italy [4] or UK [34], but no stable directives have been released yet. However, the final implementation is not expected to be significantly different, except possibly for limitations on the geographical localization and extension of the EC.

In this subsection we provide the business model background for the proposed methodology and we clarify the possible roles of aggregators and ESCOs in the context of ECs, as sketched in Fig. 1 and Fig. 2.

\subsection{Main challenges for the formation of an Energy Community}

The incentive of forming ECs is typically based on the awarding of an economic benefit when energy is produced and consumed among users of the EC [4, 34], thereby lessening the stress on the grid placed upstream. Currently, the Italian Authority is promoting ECs by means of a Feed-in-Tariff-based mechanism, similarly to UK till 2019, but other innovative models are expected to promote the coordination with local Distributor System Operators, producers and consumers [34]. This is the reason why in the proposed schemes of Fig. 1 and Fig. 2 an economic benefit is rewarded to the entity "Energy Community".

The current trend in EC adoption - e.g., in the UK, where the business figure of intermediaries seems to emerge [34] - indicates that the creation of an EC requires a number of skills that rarely belong to a local 
group of citizens whose main activities are not energy-related. Hence, the optimal design and operation of the system requires additional skills that may not be present within the EC; this will only be more pressing in the foreseeable future when innovative business models will emerge. Thus, these activities will have to be externalized to an aggregator or ESCO (from now on, simply denoted as "the aggregator" for the sake of expediency) by means of specific service contracts, as shown in Fig. 1 and Fig. 2. In this view, aggregators will be a fundamental support for ECs, provided that their goals are aligned with the ones of the components of the EC; failure to ensure this would result in the well-known "agency problem" [44].

A further challenge for the creation of the community lays in the responsibility and ownership of the assets operated in the EC. Firstly, the new installation of assets implies upfront costs that some users may not be able or willing to pay. When affordability is the issue, leasing or loans are viable options, despite being typically more expensive due to the intermediary costs. However, all the users' contributions, be them economic or other, such as availability for lending space to install assets, shall be accounted for in the distribution of the economic benefit awarded to the EC entity with efficient, rational and fair allocation criteria [46, 37, 47]. Secondly, assets may be installed at a consumer property, but the ownership may be of the consumer itself, the EC or a third company lending the asset for a fee. When the consumer does not own the asset, clauses for addressing how upfront costs are shared and how any issue possibly occurring along the lifetime of the project is solved should be put in place. This is especially critical when assets belonging to the community are installed at consumers' places. As shown in Fig. 1 and Fig. 2, assets may be installed also in locations owned by the EC for the benefit of the community; in this case the EC bears the risks of their ownership, supervision and management with respect to the other users, especially considering the diverse nature and specific contribution of the users and their aims.

In our work we primarily consider the case when the assets of the EC are owned (or lent) by the users and installed at their properties. This is considered as a flexible option because each user will be responsible of their assets and is directly motivated to maximize their return. Anyway, the proposed approach can easily be extended to tackle system configurations where part of the assets are owned by the EC.

\subsection{Externalizing sizing and operations}

As previously discussed, an EC may or may not externalize parts of the strategical and/or operational decisions to an external aggregator, with different degrees of involvement. In the simplified approach, shown in Fig. 1, the EC may only ask for support in the initial design of the system. In this case, a consulting company provides the analysis and technical support for the sizing of the assets by means of a service contract remunerated by a fee, but the main commitment ends after the installation of the assets, in case with some maintenance. In this case, assets are directly monitored and operated by the EC itself, typically by means of cheap remote controlling devices. However, the technical expertise within the EC may be limited, thereby leading to only reaping a part of the possible benefits.

A different approach, pictured in Fig. 2, is the one in which the EC delegates both the design and the operation/maintenance services to an external company. In this case, the aggregator proposes the optimal design of the system, to be approved by the EC; then, after the assets installation, it monitors and operates the system according to the clauses and rewarding scheme of the service contract. The aggregator has the technical skills necessary to maximize the benefits to the EC, but the contract and the rewarding system shall guarantee that the goal of the aggregator is aligned to that of the EC, not to incur in the agency problem. 


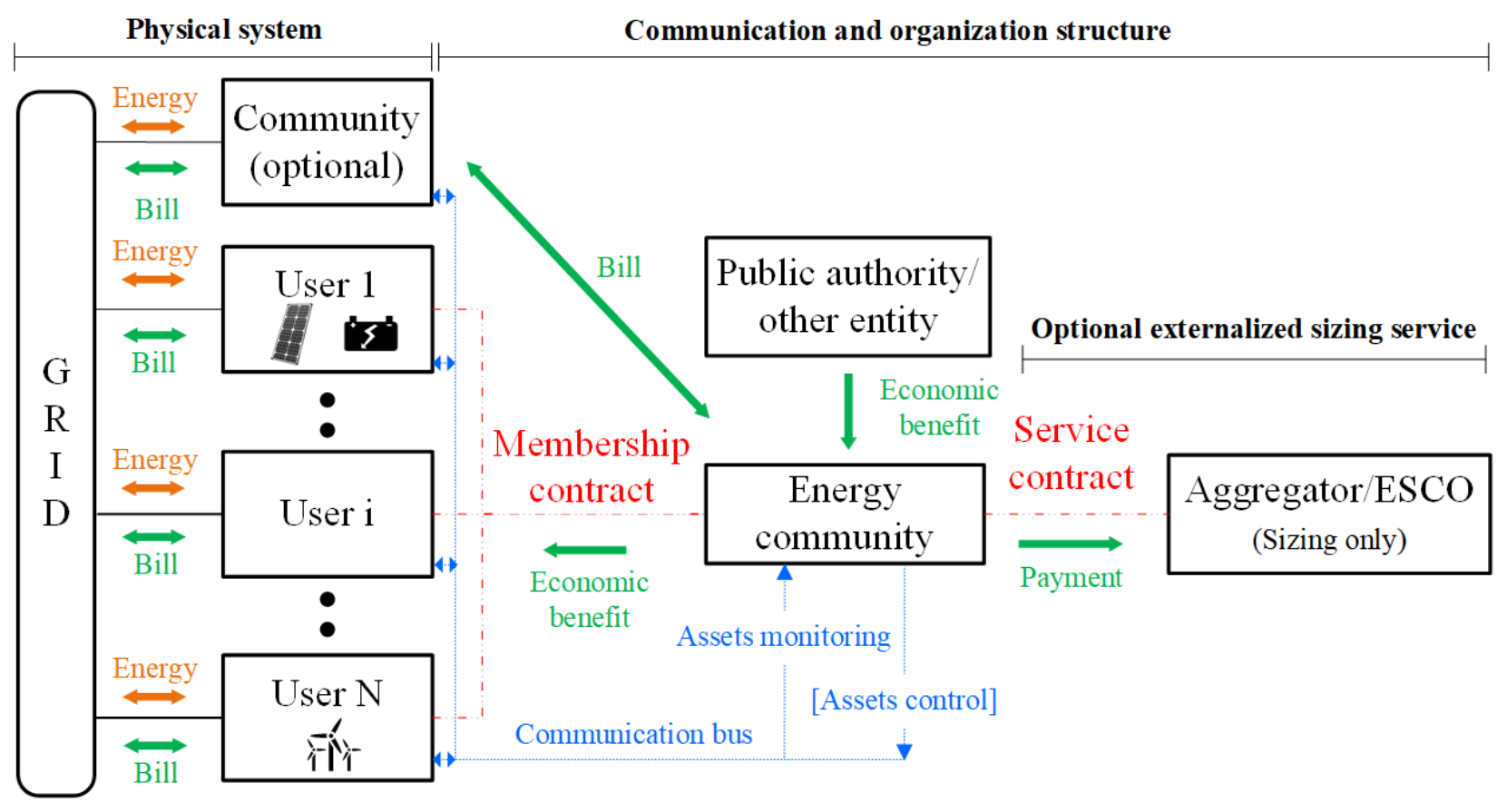

Figure 1: Business model of the energy community with no externalized activity of system operation.

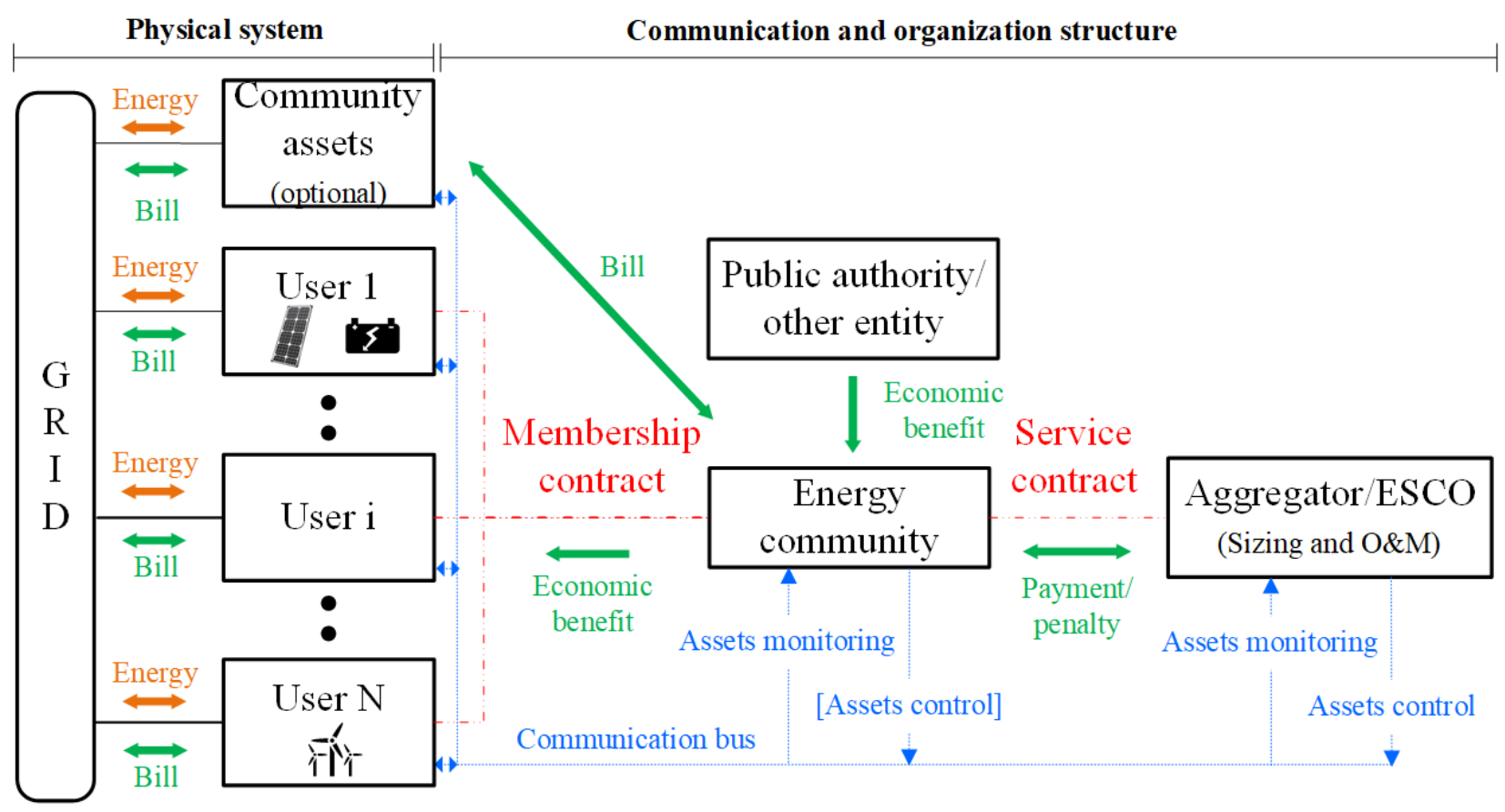

Figure 2: Business model of the energy community with externalized design and operation.

In order to maximize the potential benefits to the EC, we decided to study an externalized approach. To avoid the risks related to the agency problem, the reward for the aggregator is defined as a fraction of the economic benefit obtained by the users of the EC, as explained in the subsequent section. 


\section{The business model of the Energy Community and the aggre- gator}

\subsection{Key features of the proposed business model}

We propose an externalized business model (see Fig. 2) where:

1. users join the EC by means of membership contracts;

2. the EC may economically support users in funding new installations, but installed assets at each user property, be them in full property or on lease, are full responsibility of the user;

3. in the membership contract, exit clauses specify that any user leaving the aggregate shall reimburse the EC up a given economic amount, varying with the exit year;

4. the membership contract enables the EC and its technical advisors to access the real-time consumption data and to control some of the user's devices (e.g., batteries), which implies that proper remote communication systems are set in place at the users' premises;

5. the EC delegates the optimal design, maintenance and operation to an aggregator by means of a service contract with clauses that reduce the risk of the agency problem, as later detailed;

6. each user buys on the public market the electricity absorbed from the grid, and is paid from the public market for his extra production injected into the grid;

7. the benefits of the aggregate are rewarded to the EC that distributes them between the aggregator and the users according to a fair scheme, as discussed in Section 4.

\subsection{Service contract to avoid the agency problem}

In order to determine the best possible sizing of the system, the aggregator solves a mathematical optimization problem succinctly, described in (1).

$$
\max \left\{N P V^{A} \text { s.t. } \quad \text { Agg./Users constraints, } S W^{U, C O} \geq S W^{U, N C}\right\}
$$

The objective is maximizing its own return $N P V^{A}$ while accounting for users constraints, and in particular the fact that the social welfare $S W^{U, C O}$ of the EC, i.e., the sum of the users' NPV $\left(N P V_{i}^{U}\right)$, must be no lower than without the aggregator $\left(S W^{U, N C}\right)$. Depending on the rewarding scheme, (1) may not lead to maximize the users' social welfare, as represented by the mathematical optimization problem: the aggregator shall provide the users with a system configuration that is better than without him $\left(S W^{U, C O} \geq S W^{U, N C}\right)$, but this value may not be the global optimum for the aggregate.

$$
\max \left\{S W^{U}=\sum_{i \in I} N P V_{i}^{U} \text { s.t. } \quad \text { Users constraints }\right\}
$$

For instance, were the aggregator be rewarded with a constant fee $\left(N P V^{A}=\right.$ const $)$, it would have lower incentives to design and operate the system to maximize the real EC welfare; in other words, any feasible solution of (1) would be optimal, contrary to the EC's will. To avoid this issue, for the aggregator we propose 
a rewarding schemethat is proportional to the benefit obtained by the community with respect to the case where the aggregator was not in place $\left(S W^{t o t, N C}\right)$, as shown in (3) where $\sigma>0$ is a constant parameter.

$$
N P V^{A}=\sigma\left(S W^{U, C O}-S W^{U, N C}\right)
$$

By doing so, the aggregator objective is an affine function with respect to the objective of (2), which means that (1) achieves the same solution as (2), provided that the aggregators constraints are not restrictive with respect to the users' ones.

\subsection{The No-Assets (NA), Non-Cooperative (NC) and Aggregated Non-Cooperative (ANC) configurations}

In order to gauge the effect of an aggregator following our proposed business model, we define three possible alternative configurations. In the No-Asset (NA) configuration no user installs any assets, and therefore all the energy needs are met by buying all electricity from the public market, without any EC intervention. Since the corresponding energy bill can be very expensive, users may rationally prefer to install assets in their properties with the goal of maximizing their own profits, still without any EC being in place. In the corresponding Non-Cooperative (NC) configuration each user is independent and operates its own assets at its maximum profitability, regardless of the others; this is the configuration that we have chosen as base case scenario for the numerical simulations. It is worth mentioning that that we assume that each user, and therefore also the EC, is expected to be able to optimally size the system in this non-cooperative way, possibly with an external advisory.

When an EC is put in place the optimal collective solution can be more profitable than with the NC approach, since mutual energy exchanges within the EC yield economic benefits. Thus, users optimized independently in a $\mathrm{NC}$ way can still rationally decide to form an EC to benefit from the this additional reward without changing their behaviour. We denote as Aggregated Non-Cooperative (ANC) an EC that is formed on the same technical solution of the NC case, i.e., without any increase in the coordination of users' systems. It is worth remarking that the corresponding unintended margin is related to the enabling policy framework of the EC, as discussed in details in the following.

\subsection{The exit clause}

The combined optimization of the entire EC as a whole enables achieving higher profits than the NC and ANC configurations, but creates mutual constraints. That is, if a user leaves the EC the other members may not achieve the expected profitability targets. We propose the use of economic "exit" clauses in the membership contract whereby users are obliged to pay a time-decreasing fee in the event they leave the aggregate. These clauses are taken into account during the development of the mathematical model (cf. Section 5) and we provide a methodology to quantify the exit fee so as to ensure that the remaining users are not damaged.

\section{Game-theoretic reward allocation}

In this section we describe some game-theoretic criteria that can be used to fairly distribute the total EC reward among the users and with the aggregator (if any). 


\subsection{The Core}

The "Core" is a key concept in cooperative game theory that provides the theoretical framework to evaluate the conditions for a stable distribution of collective divisible goods among the players of a given coalition $[2,18$, 12]. The issue is that in order for the coalition composed by all players - the so-called "grand coalition" - to remain stable, the reward distribution must be such that no subset of players has incentive in leaving it. The Core is the set of the possible reward distributions that satisfies the principles of rationality and efficiency. Denoting by $v(\cdot)$ the benefits generated by the coalition that shall be distributed, a profit distribution $\left[\Delta N P V_{j}^{U A}\right]_{j \in J}$ for all users of a given (grand) coalition $J$ is rational when any sub-coalition $\hat{J} \subset J$ is awarded a higher profit in the grand coalition $\left(\sum_{j \in J} \Delta N P V_{j}^{U A}\right)$ than in a separated coalition on their own $(v(J))$, i.e., when (4) holds. A distribution is efficient when the collective benefits are distributed among all players, i.e., when (5) holds. When the Core, defined by equations (4) and (5), is non-empty, the profit allocation $\triangle N P V_{j}^{U A}$ supports the creation of the grand coalition; however, it is worth noticing that the solution $\triangle N P V_{j}^{U A}$ is not necessarily unique [12].

$$
\begin{array}{ll}
\sum_{j \in \hat{J}} \Delta N P V_{j}^{U A} \geq v(\hat{J}) & \forall \hat{J} \subseteq J \\
\sum_{j \in J} \Delta N P V_{j}^{U A}=v(J) &
\end{array}
$$

\subsection{Shapley Value (Sh)}

While a distribution in the Core guarantees the stability of a coalition, not all the - potentially infinitesolutions can generally be considered fair. Shapley [43, 9] proposed an axiomatic definition of fairness that, besides efficiency (5), also includes symmetry, additivity and dummy player. Symmetry specifies that players providing the same contribution should be rewarded with the same amount, i.e., (6). Additivity, described by (7), specifies that when two games, having characteristic functions $v$ and $w$, are added on the same players, their profit distribution shall be added. Finally, the "dummy player" property (8) specifies that a player that does not create any value for the aggregate shall be rewarded with no profit. Shapley proved that the "Shapley value" distribution is unique and described by (9). The function distributes profits with respect to the marginal contribution $v(\hat{J})-v(\hat{J} / j)$ of each user $j$ to any coalition $\hat{J}$ that can be formed within the users of the grand coalition $J$. Therefore, users that lead to higher marginal contribution tend to be rewarded more than those contributing less to the aggregate, as they belong in a larger number of sub-coalitions that create higher benefits.

$$
\begin{gathered}
v(\hat{J} \cup\{i\})=v(\hat{J} \cup\{j\}), \forall \hat{J} \subseteq J /\{i, j\} \rightarrow \Delta N P V_{i}^{U A}=\Delta N P V_{j}^{U A} \\
\Delta N P V_{j}^{U A}(v+w)=\Delta N P V_{j}^{U A}(v)+\Delta N P V_{j}^{U A}(w) \quad \forall j \in J \\
v(\hat{J} \cup\{j\})=v(\hat{J}), \forall \hat{J} \subseteq J /\{j\} \rightarrow \Delta N P V_{j}^{U A}=0 \\
N P V_{j}^{U A / A}=\frac{1}{|J|} \sum_{\hat{J} \subseteq J}\left(\begin{array}{c}
|J|-1 \\
|\hat{J}|
\end{array}\right)^{-1}[v(\hat{J})-v(\hat{J} / j)] \quad \forall j \in J
\end{gathered}
$$

A drawback of this formulation is that the explicit computation of the Shapley value via (9) require the evaluation of an exponential number of quantities and therefore can be very costly as the size of $J$ increases; however, approximation techniques have been developed for scaling the approach to large numbers [35, 28]. Furthermore, the Shapley value may not respect the rationality property, and therefore it may not belong to the Core. 


\subsection{The Nucleolus (Nuc)}

Differently from the Shapley value, the Nucleolus concept has been proposed to strengthen the stability of a coalition by distributing the profits so as to iteratively increase the total utility of the sub-coalition $J$ with the smallest surplus [42,18]. Let $\theta_{J}=\sum_{j \in J} \Delta N P V_{j}^{U A}-v(J)$ be the measure of the economic surplus that users of a sub-coalition $J$ have if they withdraw from the grand coalition; note that when $\triangle N P V^{U A}$ belongs to the Core, $\theta_{J}$ is non-negative by (4). In the Nucleolus allocation mechanism, the objective is to distribute the profits by iteratively maximizing $\theta_{J}$ for the coalition $J$ with the minimum value of the surplus. This is equivalent to progressively minimize the unhappiness of the most unhappy coalition in every iteration. The mathematical representation of the above principle is described in (10), where $\Gamma$ is the set of sub-coalitions $J \subset I$ already visited by the algorithm and $\theta_{J}$ is the value of the surplus previously fixed for the sub-coalition $J \in \Gamma$ when it had been processed in a previous iteration.

$$
\max \left\{\theta \quad \text { s.t. } \sum_{j \in J} \Delta N P V_{j}^{U A}-v(J) \geq \theta \quad \forall J \notin \Gamma, \quad \sum_{j \in J} \Delta N P V_{j}^{U A}-v(J) \geq \theta_{J} \quad \forall J \in \Gamma\right\}
$$

It can be proven that this approach leads to a unique solution when a solution exists but, similarly to the Shapley value, it involves the solution of a number of optimization problems that grows exponentially with the size of the community, being proportional to the number of possible sub-coalitions of $I$. Moreover, the Nucleolus does not necessarily satisfy the fairness axioms postulated by Shapley and therefore it is arguable whether it provides a fair distribution of profits.

\subsection{Shapley-Core (ShC)}

In our setting, the stability of the grand coalition has a paramount importance: the aggregator has no way to coerce the users to join the EC, and therefore must ensure that no user ever has a rational incentive not to join. However, fair distribution of the profits is also relevant in that, as discussed in our computational section, properly remunerating users for the contribution they give to the EC leads to an incentive to investing in assets and ultimately to an increase of the EC social welfare. We will therefore impose stability of the grand coalition, and try to produce a distribution that is "as fair as possible" under the constraint that it is stable. We propose to obtain this by using, as the reward distribution, the point in the Core closest to the Shapley distribution $\triangle N P V^{U A, S h}$, i.e., the solution of the optimization problem

$$
\min \left\{\sum_{j \in J}\left(\Delta N P V_{j}^{U A}-\Delta N P V_{j}^{U A, S h}\right)^{2} \quad \text { s.t. } \quad(4)-(5)\right\}
$$

The solution of (11) is in principle not more costly than computing $\triangle N P V^{U A, S h}$ in the first place. Furthermore, since $\triangle N P V^{U A, S h}$ is unique and the objective function in (11) is strongly convex, the resulting "Shapley-Core value" is unique as well.

\subsection{MinVariance/Core (VarC)}

The proposed Shapley/Core method is also compared with the MinVar reward distribution discussed in [1], where profits are distributed with the objective of minimizing the uneven treatment of users, while guaranteeing the solution to belong to the Core. The corresponding mathematical formulation is denoted in (12), where the objective function is the minimization of the variance of the benefit distribution, whereas the 
constraints are as in the Shapley/Core case (11).

$$
\min \left\{\sum_{j \in J}\left(\Delta N P V_{j}^{U A}-\frac{1}{|J|} \sum_{j \in J} \Delta N P V_{j}^{U A}\right)^{2} \quad \text { s.t. } \quad(4)-(5)\right\}
$$

The main advantage of this formulation is that it does not involve the calculation of the Shapley function, thus (possibly with the help of decomposition techniques) the entire technical and reward distribution problems solution may be faster to compute $[1,32]$. On the other hand, while belonging to the Core the final distribution may privilege users only because their absolute NPV value may be larger than others, whose fairness properties may be questionable.

\subsection{Shapley-Nucleolus (ShNuc)}

Although the Shapley/Core approach introduced in Section 4.4 enables achieving a stabilized Shapley-like reward distribution, we cannot guarantee that each coalition has a strictly positive surplus: in fact, some of the constraints defining the core may hold with equality, thus leading to a null surplus. That means that a sub-coalition is as profitable in the grand coalition as on their own, which does not go in favor of stability.

Therefore, in order to strengthen the stability, we also propose a modified Shapley/Nucleolus distribution where first the utility of the coalition with the smallest surplus is maximized, then the profits are distributed "as fair as possible", similarly to the Shapley/Core case. The corresponding mathematical problem is reported in (13), where $\bar{\theta}$ represents the surplus of the most unhappy coalition, obtained by solving (10).

$$
\min \left\{\sum_{i \in J}\left(\Delta N P V_{j}^{U A}-\Delta N P V_{j}^{U A, S h}\right)^{2} \quad \text { s.t. } \quad \sum_{j \in J} \Delta N P V_{j}^{U A}-v(J) \geq \bar{\theta},(5)\right\}
$$

\subsection{MinVariance/Nucleolus (VarNuc)}

Similarly to the Shapley/Nucleolus case, also in the case of the the MinVariance/Core mechanism there might be coalitions whose surplus is null. Therefore, we also propose the equivalent MinVariance/Nucleolus function where first the utility of the most unhappy coalition is maximized, then the variance of the benefit distribution is minimized. The mathematical formulation is reported in (14).

$$
\min \left\{\sum_{j \in J}\left(\Delta N P V_{j}^{U A}-\frac{1}{|J|} \sum_{j \in J} \Delta N P V_{j}^{U A}\right)^{2} \quad \text { s.t. } \quad \sum_{j \in J} \Delta N P V_{j}^{U A}-v(J) \geq \bar{\theta},(5)\right\}
$$

\section{$5 \quad$ Mathematical Model}

\section{$5.1 \quad$ Structure}

In this section, we detail the main optimization procedure used to:

1. optimal size and operate the users' system both in a non-cooperative (cases NA, NC and ANC) and cooperative (case CO) configurations;

2. fairly allocate the total reward awarded to the EC among the users, for the ANC and CO configurations;

3. calculate the exit costs for ANC and CO.

The methodology described in the following subsections is aimed at providing a solid understanding of the main technical and economic constraints for sizing ECs with the objective of (1) providing an optimization 
method for ECs based on the proposed business model, (2) evaluating advantages and drawbacks of the selected game-theoretic reward allocation functions, and (3) calculating the exit costs. In particular, as clarified in the following, these three main problems are decoupled and can be solved in a row: first (1), then (2) and finally (3). This characteristic of the problem dramatically simplifies the solving strategy and therefore considerably enhances the practical usefulness of the proposed approach.

In particular, to perform task 1, cost parameters at the user place and adequate forecasts of both the load demand and renewable energy production for the selected users are needed. In the NA, NC and ANC cases, those assessments are performed by each user independently, if necessary with the support of a delegate; conversely in the $\mathrm{CO}$ case a central entity like an aggregator shall receive enough information to do so. In the latter case, as discussed in Section 3, users' privacy can be addressed by legal agreements that forbid the aggregator to misuse the private information, thus that requirement is not expected to be a significant barrier for the EC creation.

To properly estimate the operation costs, the model accounts for the seasonality in the load profile, the renewable energy production and their variability along the year. In particular, the yearly simulations are approximated by using representative days so as to guarantee a good compromise between accuracy of the results and low computational cost. According to the suggestion of [39], in this study 12 representative days are selected to capture the main variations in both the load and renewable production power profiles.

\subsection{Optimal design and sizing model}

In this section, we introduce the main techno-economic modeling of the optimization of the users' energy system and the aggregate for the different configurations under consideration: No-Assets (NA), Non-Cooperative (NC), Aggregated Non-Cooperative (ANC) and COoperative (CO).

\subsubsection{User objective}

In the NA, NC and ANC configurations, each single user $j \in J$ aims at maximizing its own Net Present Value (NPV), shown in (15), that does not account for any reward by the EC, since no coordination is in place. The NPV accounts for the net economic balance $R_{j, y}^{U, P}$ between energy sold and bought from the public market, the costs $C_{j, y}^{U, P}$ due to the peak power, the investment costs $C A P E X_{j, y}^{U}$, the replacement of the assets $R C_{j, y}^{U}$ and their residual value $R V_{j, y}^{U}$ at the end of the project lifetime $|Y|$, taking into account the discount factor $d$.

$$
\max N P V_{j}^{U}=\sum_{y \in Y} \frac{\left[R_{j, y}^{U, P}-C_{j, y}^{U, P}-C A P E X_{j, y}^{U}-R C_{j, y}^{U}+R V_{j, y}^{U}\right]}{(1+d)^{y}}
$$

The net economic balance due to the energy exchanged with the public grid is shown in (16). Be the EC in place or not, every unit of energy drained by the user at its own POD, corresponding to a non-null power dispatch of $P_{j, t}^{U, P O D-}$, is bought from the public market at the national price $\pi_{t}^{P-, V}+\pi_{t}^{P-, F}$, where $\pi_{t}^{P-, F}$ is the tariff component on the demand consumption and $\pi_{t}^{P-, V}$ is the tariff on the withdrawing. The same applies when energy is sold, yet with power dispatch $P_{j, t}^{U, P O D+}$ and price $\pi_{t}^{P+}<\pi_{t}^{P-, V}$. Accordingly, the peak power $\operatorname{cost} C_{j, y}^{U, P}$ is proportional to the maximum power exchange $P_{j, w}^{U, \text { max }}$ at the POD, be it inwards or outwards. As representative days are used to characterize the yearly load demand, the coefficients $m_{t}^{T}$ and 
$m_{w}^{W}$ are used to normalize the values at yearly horizon; $\Delta$ is the time resolution (15-min).

$$
\begin{array}{ll}
R_{j, y}^{U, P}=\Delta \sum_{t \in T} m_{t}^{T}\left[\pi_{t}^{P+} P_{j, t}^{U, P O D+}-\pi_{t}^{P-, V} P_{j, t}^{U, P O D-}-\pi_{t}^{P-, F} P_{j, t}^{L}\right] & \forall y \in Y / 0 \\
C_{j, y}^{U, P}=\sum_{w \in W} m_{w}^{W} c_{w}^{P P} P_{j, w}^{U, \max } & \forall y \in Y / 0
\end{array}
$$

The investment costs $C A P E X_{j, y}^{U}$, occurring only at the initial year $(y=0)$, are proportional to the installed capacity $x_{j}^{a, U}$ of each component $a$ with given coefficient $c_{j}^{a, U}$. The maintenance charges $C_{j, y}^{U, M}$ are proportional to the installed capacity by the coefficient $c_{j}^{a, U, M}$, similarly to CAPEX. The replacement costs $R C_{j, y}^{U}$ are accounted for in (20): when a component reaches its end of life $N_{j}^{Y, a}$, it is replaced with a new one. Moreover, at the end of the project $(y=|Y|)$ the residual value of all components $R V_{j, y}^{U}$ is evaluated.

$$
\begin{aligned}
& C A P E X_{j, y}^{U}=\sum_{a \in A_{j}} x_{j}^{a, U} c_{j}^{a, U} \quad y=0 \\
& C_{j, y}^{U, M}=\sum_{a \in A_{j}} x_{j}^{a, U} c_{j}^{a, U, M} \quad \forall y \in Y / 0 \\
& R C_{j, y}^{U}=\sum_{a \in A_{j}}\left\{\begin{array}{ll}
x_{j}^{a, U} c_{j}^{a, U} & \bmod \left(y, N_{j}^{Y, a}\right)=0 \\
0 & \text { else }
\end{array} \quad \forall y \in Y / 0\right. \\
& R V_{j, y}^{U}=x_{j}^{a, U} c_{j}^{a, U} \frac{N_{j}^{Y, a}-\bmod \left(|Y|-1, N_{j}^{Y, a}\right)}{N_{j}^{Y, a}} \quad \forall y=|Y|
\end{aligned}
$$

\subsubsection{Aggregator objective}

The aggregator, instead, aims at maximizing its own Net Present Value $\left(N P V^{A}\right)$ that, as specified by the service contract in the proposed business model in Section 3, is mathematically modelled in (22) as a fraction $\sigma$ of the benefit gained by the users $\left(S W^{U, C O}(I \cup A)-S W^{U, N C}(I)\right)$. The social welfare of the users aggregated by the aggregator $S W^{U, C O}(I)$ is the difference between the overall social welfare $S W^{t o t, C O}(J \cup A)$ and the payment to the aggregator $N P V^{A}$, detailed in (23). It is worth noticing that the total social welfare of the users is then distributed among them according to fair rules, described in the forthcoming sections; the final NPV of the users accounting for the reward allocation is denoted as $N P V_{j}^{U A}$ in (23). $S W^{t o t, C O}(J \cup A)$ accounts for all revenues and expenses for users, energy community and aggregator, thus it is the sum of the $\operatorname{NPV}\left(N P V_{j}^{U}\right)$ of the users as defined in Section 5.2.1 and the reward $E C R_{y}(J)$ allocated to the EC by the public entity, which is a fraction of the energy shared among the users, defined in (25). To do so, the power scheduling at the POD of each user is divided in terms of the quantity that contributes to the shared energy $P_{j, t}^{U, M+/-}$ and the residual quantity $P_{j, t}^{U, P+/-}$, see (26).

$$
\begin{aligned}
& \max N P V^{A}(I \cup A)=\sigma\left[S W^{U, C O}(I \cup A)-S W^{U, N C}(I)\right] \\
& S W^{U, C O}(J \cup A)=\sum_{j \in J} N P V_{j}^{U A}=S W^{t o t, C O}(J \cup A)-N P V^{A}(J) \quad \forall J \subseteq I \\
& S W^{t o t, C O}(J \cup A)=\sum_{j \in J} N P V_{j}^{U}+\sum_{y \in Y / 0} \frac{E C R_{y}(J)}{(1+d)^{y}} \quad \forall J \subseteq I \\
& E C R_{y}(J)=\sum_{t \in T} m_{t}^{T} \pi_{t}^{R} E_{t}^{S H} \quad \forall J \subseteq I, y \in Y / 0 \\
& P_{j, t}^{U, P O D+/ P O D-}=P_{j, t}^{U, P+/ P-}+P_{j, t}^{U, M+/ M-} \quad \forall j \in J \subseteq I, t \in T
\end{aligned}
$$

According to the formulation detailed in (22) and (23), it is easy to verify with simple algebraic manipulations that $N P V^{A}(I \cup A)$ can be expressed as an affine function with respect to the social welfare of the 
entire system $\left(S W^{t o t, C O}(J \cup A)\right)$, as shown in $(27)$.

$$
N P V^{A}(J \cup A)=\frac{\sigma}{1+\sigma} S W^{t o t, C O}(J \cup A)-\frac{S W^{U, N C}(J)}{1+\sigma} \quad \forall J \subseteq I
$$

In fact, both $\sigma$ and $S W^{U, N C}(I)$ are constants for the problem of the aggregator. Moreover, since the remuneration of the aggregator cannot but be a positive fraction of the total generated profits, $\sigma$ is a strictly positive constant, which means that the solution obtained by maximizing $N P V^{A}(I \cup A)$ is equivalent to one obtained by maximizing over the social welfare of the aggregate $\left(S W^{t o t, C O}(J \cup A)\right)$. This confirms that the proposed business model enables aligning the optimization problem of the aggregator towards the maximization of the social welfare for the entire system, which goes in favor of addressing the potential agency problem.

\subsubsection{User technical constraints}

The main technical constraints related to the internal system of each user account for the power and energy balance and capabilities of the components as follows. These equations hold for each user $j \in J /\{A\}$ in all the configurations under test (NA, NC, ANC and $\mathrm{CO}$ ).

The power balance at each internal system is guaranteed by (28), where $P_{j, t}^{U, P O D+/-}$ is the power dispatch at the user's POD, $P_{j, t}^{c+/-, U}$ represents the power dispatch of the battery converter ( "+" when supplying, “-" when absorbing), $P_{j, t}^{R, U}$ is the renewable production, and $P_{j, t}^{L}$ represents the load demand.

$$
P_{j, t}^{U, P O D+}-P_{j, t}^{U, P O D-}+\sum_{c \in A_{j}^{C}}\left[P_{j, t}^{c-, U}-P_{j, t}^{c+, U}\right]-P_{j, t}^{R, U}=-P_{j, t}^{L} \quad \forall t \in T
$$

The peak power at the user POD is calculated with (29) and (30). (31) specifies the maximum renewable power dispatch available at every time step for every user; $x_{j}^{r, U}$ represents the installed capacity of the renewable asset $r$ and $p_{j, t}^{r, U}$ is its specific power production.

$$
\begin{array}{lr}
P_{j, w}^{U, \max } \geq P_{j, \hat{t}}^{U, P O D+}-P_{j, \hat{t}}^{U, P O D-} & \forall w \in W, \hat{t} \in \hat{T}_{w} \\
P_{j, w}^{U, \max } \geq-\left[P_{j, \hat{t}}^{U, P O D+}-P_{j, \hat{t}}^{U, P O D-}\right] & \forall w \in W, \hat{t} \in \hat{T}_{w} \\
P_{j, t}^{R, U} \leq \sum_{r \in A_{j}^{R}} p_{j, t}^{r, U} x_{j}^{r, U} & \forall t \in T
\end{array}
$$

The energy balance in the batteries is instead modeled by (32) and (33), for a generic time step and the initial one, respectively. In the equations, the roundtrip efficiency $\eta_{j}^{b}$ of the battery system $b$, including its converter $c(b)$, is accounted for. The peak power capacity of the converter is guaranteed by (34), whereas the maximum and minimum state of charge of the battery are specified in (35) by coefficients $\beta_{j}^{b, \max / \min } \cdot x_{j}^{b, U}$ represents the energy capacity of the battery $b$ and $x_{j}^{c(b), U}$ is the power capacity of the converter corresponding to battery $b$.

$$
\begin{array}{lr}
E_{j, t}^{b, U}=E_{j, t-1}^{b, U}-\Delta P_{j, t}^{c(b)+, U} / \sqrt{\eta_{j}^{b}}+\Delta P_{j, t}^{c(b)-, U} \sqrt{\eta_{j}^{b}} & \forall b \in A_{j}^{B}, t \in T / 1 \\
E_{j, 1}^{b, U}=E_{j,|T|}^{b, U}-\Delta P_{j, 1}^{c(b)+, U} / \sqrt{\eta_{j}^{b}}+\Delta P_{j, 1}^{c(b)-, U} \sqrt{\eta_{j}^{b}} & \forall b \in A_{j}^{B}, t=1 \\
P_{j, t}^{c, U}+P_{j, t}^{c-, U} \leq x_{j}^{c, U} & \forall c \in A_{j}^{C}, t \in T \\
x_{j}^{b, U} \beta_{j}^{b, \text { min }} \leq E_{j, t}^{b, U} \leq x_{j}^{b, U} \beta_{j}^{b, \max } & \forall b \in A_{j}^{B}, t \in T
\end{array}
$$


Finally, bounds (36)-(43) on the variables are specified to make the problem tighter and faster to compute.

$$
\begin{array}{lr}
x_{j}^{a, U} \leq \bar{x}_{j}^{a, U} & \forall a \in A_{j} \\
P_{j, t}^{c+, U} \leq \bar{x}_{j}^{c, U} & \forall c \in A_{j}^{C}, t \in T \\
P_{j, t}^{c-, U} \leq \bar{x}_{j}^{c, U} & \forall c \in A_{j}^{C}, t \in T \\
P_{j, t}^{R, U} \leq \sum_{r \in A_{j}^{R}} \bar{x}_{j}^{r, U} & \forall t \in T \\
E_{j, t}^{b, U} \leq \bar{x}_{j}^{b, U} & \forall b \in A_{j}^{B}, t \in T \\
P_{j, t}^{U, M / P+} \leq \sum_{c \in A_{j}^{C}} \bar{x}_{j}^{c, U}+\max _{t \in T}\left[\sum_{r \in A_{j}^{R}} p_{j, t}^{r, U} \bar{x}_{j}^{r, U}\right]-\min _{t \in T} P_{j, t}^{L}=\bar{P}_{j, t}^{U+} & \forall t \in T \\
P_{j, t}^{U, M / P-} \leq \max _{t \in T} P_{j, t}^{L}+\sum_{c \in A_{j}^{C}} \bar{x}_{j}^{c, U}-\min _{t \in T}\left[\sum_{r} p_{j, t}^{r, U} \bar{x}_{j}^{r, U}\right]=\bar{P}_{j, t}^{U-} & \forall t \in T \\
P_{j, w}^{U, m a x} \leq \max _{\hat{t} \in \hat{T}_{w}}\left\{\bar{P}_{j, \hat{t}}^{U-}, \bar{P}_{j, \hat{t}}^{U+}\right\} & \forall w \in W
\end{array}
$$

\subsubsection{EC constraints}

In the cooperative configurations ( $\mathrm{ANC}$ and $\mathrm{CO}$ ), the commercial power balance of the energy exchanged between the users is expressed by (44), where $E_{t}^{S H}$ identifies the energy shared within the EC.

$$
\sum_{j \in J} P_{j, t}^{U, M+}=\sum_{j \in J} P_{j, t}^{U, M-}=E_{t}^{S H} / \Delta \quad \forall t \in T
$$

\subsubsection{Problems definition for the NA, NC, ANC and CO problems}

Given the model of the system detailed above, the optimal design and operation of the Non-Cooperative (NC) solution is obtained by solving the problem described in (45) independently for the each user. Since each user solves its own problem, the total number of problems solved is $|I|$.

$$
\max \left\{N P V_{j}^{U}[\text { See (15)-(21)] s.t. } \quad(28)-(43)\}\right.
$$

The problem of the No-Assets (NA) configuration corresponds to solving the NC problem in (45) where users cannot install any asset, i.e., $x_{j}^{a, U}=0, \forall a \in A_{j}, j \in I$.

The Aggregated Non-Cooperative (ANC) configuration is based on the technical solution and optimal scheduling of the $|I| \mathrm{NC}$ problems in (45), but also accounts for the total reward obtained by the mutual energy sharing that would have occurred in the $\mathrm{NC}$ configuration anyway. The reward is calculated by definition as in (25) and the corresponding total social welfare is defined in (52). This added benefit is quantified and then distributed among the users with appropriate fair revenues shares, discussed in the next section.

Finally, in the COoperative approach (CO), the aggregator is asked to conjointly optimize both the design and operation for all users, given the remuneration scheme of the aggregator detailed in Section 3. According to Section 3 and Section 5.2.2, the original optimization problem of the aggregator is

$$
\max \left\{N P V^{A g g}[\operatorname{See}(22)-(26),(15)-(21)] \quad \text { s.t. } \quad(28)-(44)\right\}
$$

As shown in (22), the fair remuneration of the aggregator is expressed proportionally to $\sigma$, that is a key parameter for the definition of $N P V^{A g g}$ but is not known a-priori. However, as discussed in Section 5.2.2, the solution of the problem (46) where $N P V^{A g g}$ is maximized can be easily calculated by solving an equivalent problem where the total social welfare $\left(S W^{t o t, C O}(I \cup A)\right)$ is maximized. This latter formulation, detailed in 
(47), does not need a numerical value for $\sigma$, therefore it is regarded to be more appropriate for the proposed methodology. The fair retribution of the aggregator is calculated as discussed in the following sections.

$$
\max \left\{S W^{\text {tot }, C O}(I \cup A) \quad[\text { See }(24)-(26),(15)-(21)] \quad \text { s.t. } \quad(28)-(44)\right\}
$$

It is worth noticing that in the problem stated above the specific profit distribution by user does not affect the optimal design and operation of the system. This is a crucial characteristic that enables decoupling the reward allocation and the technical problems and goes in favour of the practical applicability of our approach.

\subsection{Fair reward allocation modelling}

The total reward awarded to an EC shall be properly distributed among users (ANC and CO configurations) and aggregator (only $\mathrm{CO}$ ). In order to test the game-theoretic reward allocation schemes selected and discussed in Section 4, in this subsection we provide the mathematical background to link the formulation described in Section 4 and the proposed EC problem.

\subsubsection{Notation}

First, the outcome of the reward distribution problem is the definition of the added benefit $\triangle N P V_{j}^{U A / A}$ by user and aggregator that adds up to the base case cost corresponding to the NC configuration, as specified in (48). Therefore, the aggregator distributes the reward $R E W_{j}^{U}$ to match the desired profitability of the user; in the case of the aggregator, $N P V^{A}=\triangle N P V^{A}$ holds as its NC profitability is null.

In order to calculate $\triangle N P V_{j}^{U A / A}$, the reward allocation problems in Section 4 are expressed with respect to the total benefit $v(J)$ generated by the aggregate $J$. In the EC context, this value corresponds the opportunity cost of the most profitable configuration (ANC or $\mathrm{CO}$ ) with respect to the $\mathrm{NC}$ case; this is translated into mathematical terms with (49)-(53). In particular, (49) defines the opportunity cost $v(J)$, where $S \ddot{W}^{t o t}(J)$ denotes the social welfare of any subset $J$ of players in their most profitable configuration and $S W^{N C}(J)$ represents the social welfare in the corresponding NC configuration. As described in (50), when the aggregator is included in the set $J$ and the coalition has two or more users, $S W^{t o t}(J)$ equals the social welfare $\overline{S W}^{t o t, C O}(J)$ obtained with the CO configuration over the players $J$, otherwise the ANC social welfare $S W^{A N C}(J)$ is considered. It is worth noticing that $\overline{S W}^{t o t, C O}(J)$ is calculated by solving an auxiliary CO optimization problem shown in (51) that corresponds to problem (47), but with a potentially different subset of players $J$. For the purpose of the reward distribution problem, the terms $\overline{S W}^{\text {tot,CO }}(J)$ can then be calculated by solving these auxiliary problems before the rewarding allocation problem, which makes our approach cheaper to solve. When only users are in the coalition $J$, no aggregator manages the EC and no coordinated design nor operation occurs; therefore the expected EC social welfare corresponds to the ANC case $S W^{A N C}(J)$, as reported in (52). Finally, when only a user is in the coalition, be it with or without the aggregator, $S \ddot{W}^{\text {tot }}(J)$ equals the NPV of the corresponding NC configuration. For the sake of 
comprehensiveness, we also report the social welfare of the NC case, shown in (53).

$$
\begin{aligned}
& N P V_{j}^{U A}(J)=N P V_{j}^{U, N C}(J)+\Delta N P V_{j}^{U A}(J)=N P V_{j}^{U}(J)+R E W_{j}^{U}(J) \quad \forall j \in J, J \subseteq I \cup\{A\} \\
& v(J)=S \ddot{W}^{t o t}(J)-S W^{N C}(J) \\
& \forall J \subseteq I \cup\{A\} \\
& S \ddot{W}^{t o t}(J)= \begin{cases}\overline{S W}^{t o t, C O}(J) & \{A\} \in J \wedge|J|>2 \\
S W^{A N C}(J) & \{A\} \notin J \\
N P V_{j}^{U, N C} & J=\{i, A\} \\
0 & J=\emptyset \vee J=\{A\}\end{cases} \\
& \overline{S W}^{t o t, C O}(J)=\max \left\{S W^{t o t, C O}(J) \text { s.t. (28)-(44) }\right\} \quad \forall J \subseteq I \cup\{A\}:\{A\} \in J \\
& S W^{A N C}(J)= \begin{cases}S W^{N C}(J)+\sum_{y} \frac{E C R_{y}^{A N C}(J)}{(1+d)^{y}} & |J|>1 \\
S W^{N C}(J) & |J| \leq 1\end{cases} \\
& S W^{N C}(J)= \begin{cases}\sum_{j \in J} N P V_{j}^{U, N C} & J \neq \emptyset \\
0 & J=\emptyset\end{cases} \\
& \forall J \subseteq I \cup\{A\}
\end{aligned}
$$

\subsubsection{Reward allocation problems}

Crucially, the optimal design and operation models stated in Section 5.2 are not constrained by the reward distribution problems, which means that the two problems can be decoupled and solved in sequence: first, the EC design and operation are optimized; then, the reward allocation and the exit costs are calculated accordingly.

Therefore, the optimal reward allocation problems discussed in Section 4 can be solved as-is, provided the mathematical links stated in (48)-(53). For the sake of clarity, we do not repeat the mathematical modelling of the different reward schemes, but the corresponding problems have been implemented and solved accordingly.

\subsection{Exit clauses}

Finally, equations (54) and (55) describe the procedure to calculate the value of the exit clauses, aimed at preserving the economic benefits of the EC players in the case a user leaves the aggregate, as discussed in Section 3. In particular, when a user $i$ leaves at year 1, the rest of the aggregate can only achieve the social welfare $\overline{S W}^{t o t, C O}(I / i \cup A)(I /\{i\})$, whereas before the user exited, their reward was $S W^{t o t, C O}(I \cup$ $A)-N P V_{i}^{U A}$; therefore, the user shall pay this difference $V_{i, 1}^{\text {exit }}$, namely the exit clause. $\overline{S W}^{\text {tot, } C O}(I / i \cup A)$ has already been calculated by the auxiliary problems detailed in (51) and $N P V_{i}^{U A}$ is the total user NPV accounting for a specific reward scheme, calculated with the methodology in the previous subsection.

However, it is unlikely that a user exits the EC within the first year. It is more plausible that the event occurs $y$ years after the installation, e.g. because unexpected personal needs. In that case, the exit cost $V_{i, y}^{\text {exit }}$ for the user exiting in a generic year $y$ shall decrease because the EC is affected for the remaining years of 
the project $(|Y|-y+1)$, as detailed in (55).

$$
\begin{aligned}
V_{i, 1}^{\text {exit }}=S W^{t o t, C O}(I \cup A)-N P V_{i}^{U A}-\overline{S W}^{t o t, C O}(I / i \cup A) & \forall i \in I, y=1 \\
V_{i, y}^{\text {exit }}=V_{i, 1}^{\text {exit }} \frac{\sum_{\hat{y}=1}^{|Y|-y+1} \frac{1}{(1+d)^{\hat{y}}}}{\sum_{\bar{y}=1}^{|Y|} \frac{1}{(1+d)^{y}}} & \forall i \in I, y \in Y: y>1
\end{aligned}
$$

By using the proposed exit cost, the energy community and aggregator are provided with a tool to quantify and clarify what happens in the case a user leaves the aggregate.

\section{Case Study}

The case study aims at validating the proposed business model and comparing both the different optimization methodologies (NA, NC, ANC and CO) and the different reward distribution schemes. The numerical example focuses on a possible EC composed by 10 large residential/commercial users served by a typical distribution substation in the peri-urban area of an Italian city. However, due to lack of Italian data and given their similar socio-economic behaviour, we used as a reference the electrical profiles measured from a Portuguese substation [49], with average yearly demand in the range 12-31 MWh/y. The location favour the installation of PV solar and wind resources; data about the corresponding energy output for our study has been determined according to [38]. When energy is purchased from the grid, the electricity price is assumed to be $18 \mathrm{c} \in / \mathrm{kWh}$, of which $2 \mathrm{c} € / \mathrm{kWh}$ corresponds to fixed costs $\left(\pi_{t}^{P-, F}\right)$; when energy is sold to the grid, the tariff $\left(\pi_{t}^{P+}\right)$ is $5 \mathrm{c} \in / \mathrm{kWh}$ [4]. Moreover, the EC reward price $\left(\pi_{t}^{R}\right)$ is assumed to be $10.8 \mathrm{c} € / \mathrm{kWh}$, in agreement with the present Italian regulation [4].

The main economic and technical parameters for the possible installations in the users are specified in Table 1, based on realistic cost data [29]. The lifespan of the PV and wind system are 25 and 20 years, respectively; the lithium batteries are assumed to last 15 years at $80 \%$ Depth of Discharge (DoD), and their battery converters are expected to operate for 10 years. The yearly maintenance cost of the PV and wind systems are $30 € / \mathrm{kW}$, whereas the ones of the battery and the converter are $5 € / \mathrm{kWh}$ and $2 € / \mathrm{kWh}$, respectively.

\begin{tabular}{|c|c|c|c|c|c|c|c|c|c|c|c|c|}
\hline \multirow[t]{2}{*}{ Asset } & & \multirow[t]{2}{*}{ Unit } & \multicolumn{10}{|c|}{ User } \\
\hline & & & $\mathrm{U} 1$ & $\mathrm{U} 2$ & U3 & $\mathrm{U} 4$ & U5 & $\mathrm{U} 6$ & $\mathrm{U} 7$ & U8 & U9 & $\mathrm{U} 10$ \\
\hline $\mathrm{PV}$ & Limit & $\mathrm{kW}$ & 50 & 80 & 100 & 40 & & 60 & & 100 & 100 & \\
\hline PV & CAPEX & $€ / \mathrm{kW}$ & 1700 & 1400 & 1600 & 1400 & & 1400 & & 1400 & 1400 & \\
\hline Wind & Limit & $\mathrm{kW}$ & & & 100 & & & & & 100 & & \\
\hline Wind & CAPEX & $€ / \mathrm{kW}$ & & & 3000 & & & & & 3000 & & \\
\hline Batt & Limit & kWh & & 50 & 50 & & & 50 & & 50 & 50 & \\
\hline Batt & CAPEX & $€ / \mathrm{kWh}$ & & 400 & 400 & & & 500 & & 400 & 400 & \\
\hline Conv & Limit & $\mathrm{kW}$ & & 50 & 50 & & & 50 & & 50 & 50 & \\
\hline Conv & CAPEX & $€ / \mathrm{kW}$ & & 200 & 200 & & & 200 & & 200 & 200 & \\
\hline
\end{tabular}

Table 1: Main technical and economic parameters by user.

In the results section, we describe the optimal design for the EC operated by an aggregator based on the business model described in Section 3 and its mathematical formulation detailed in Section 5. In particular, 
the cooperative model $(\mathrm{CO})$ is compared to both the non-cooperative configurations (NA, NC and ANC) to highlight the possible benefits provided by the aggregator; possible fair schemes (Shapley value, Core, Nucleolus and Core/Shapley) to distribute the reward granted to the energy community are compared.

\section{Results}

The main economic results of the proposed method are shown in Fig. 3, Fig. 6, Fig. 9, Fig. 10 and Table 3, whereas the main technical results are reported in Fig. 7, Fig. 8, Fig. 4, Fig. 5 and Fig. 11. For simplicity, since most of the numerical values of the social welfare and NPV are negative values, we refer to their opposite, namely Social Cost $(S C=-S W)$ and Net Present Cost $(N P C=-N P V)$, respectively.

\subsection{Social costs by aggregate level and user}

The social costs corresponding to the methodologies (NA, NC, ANC and $\mathrm{CO}$ ) are shown in Fig. 3; as expected, higher degrees of assets installation and coordination allow to decrease costs. In the NA case, where no assets are installed and no coordination is in place, costs peak at almost 4.2M€, whereas they drop to 3.7M€ in the Non-Cooperative case, thanks to the installed renewable generation and batteries that allow users to decrease their electricity bill. Anyway, only the CO configuration enables achieving the lowest costs, from $3 \%$ to $18 \%$ lower than any other configuration, thanks to the optimal design and operation that the aggregator can ensure and suggest to the EC. Therefore, these analyses confirm and quantify the positive economic impact of a cooperative approach enabled by the aggregator.

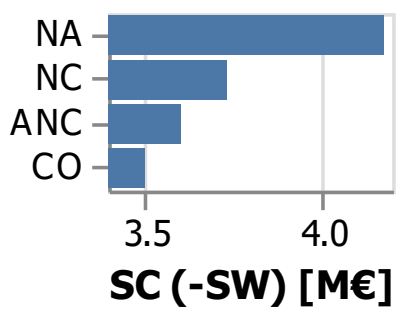

Figure 3: Social cost by configuration: No-Assets (NA), Non-Cooperative (NC), Aggregated NC (ANC) and COoperative $(\mathrm{CO})$.

Moreover, it is worth noticing that in the ANC configuration, where a non-coordinated EC is in place, social costs are 3-4\% lower than in the $\mathrm{NC}$ case, although on a technical and operational perspective the ANC and NC cases are absolutely identical. In fact, the ANC and NC cases correspond both on the size of components and on the energy flows at the user PODs; in the case of ANC, the status of EC is acknowledged and the users receive an additional economic amount that is proportional to the energy flows that naturally occurred among them. However, this - possibly substantial - benefit may in fact be an undesirable effect from the point of view of the public authority, because it is a mere incentive to prosumers/consumers that may not correspond to any additional social or technical benefit, nor to added installed assets. Therefore, this suggests that further policy and research studies on business models shall be developed to reduce the effects of this unwanted behaviour; to the best of our knowledge, this work is the first having quantitatively 
discussed and detailed this issue. Instead, coordinated approach such as the $\mathrm{CO}$ configurations should be promoted.

\subsection{Energy shares by user and aggregate type}

Fig. 4 describes the energy flows exchanged among the producers on the left-hand side and the consumers on the right-hand side for the $\mathrm{NC}, \mathrm{ANC}$ and $\mathrm{CO}$ cases; the energy bought and sold by users is also highlighted, as well as the self-consumption of each user and the energy shared within the EC, when applicable. It is worth noticing that the $\mathrm{CO}$ configuration achieves the highest self/shared consumption within the community, which is more than twice as large as in the ANC case. Indeed, the shared energy in the ANC case is limited and accidental since it depends on the optimal sizing and operation of the NC case, that is independent for each user and not designed to improve shared consumption. It is worth noticing that the difference between the NC and ANC cases, Fig. 4a and Fig. 4b, respectively, lays only in the formation of the block the self/shared energy denoted with the quantity "community", which also enables reducing the energy bought and sold from the grid. However, this difference is only at a commercial level: in the ANC and NC cases the energy flows at the user PODs are identical, but in the ANC case the EC framework enables accounting for the self/shared energy, contrarily to the $\mathrm{NC}$ case. These pictures further confirm the beneficial effects of the solution coordinated by the aggregator in terms of shared energy, which goes in favor of the social and environmental goals of the EU policies on ECs.

Fig. 5 focuses on energy sharing in the perspective of each single user, in any tested case. The total dependence on the external grid in the CO case is only $54 \%$, which means about $46 \%$ of self/shared consumption, whereas in the NC/ANC configurations public market amounts to $66-72 \%$ of energy exchanges, which means only $28-34 \%$ self/shared consumption. However, the total penetration of renewable sources increases from $35 \%$ in $\mathrm{NC} / \mathrm{ANC}$ to $52.5 \%$ in $\mathrm{CO}$, which is higher than the self/shared consumption (46\%), since a fraction of energy is directly sold to the grid, as shown in Fig. 4c. This suggests that the main benefits are related to increased values of energy shared among users, rather than in terms of renewable production itself, which goes in favour of promoting positive attitudes between the users. In particular, when comparing the ANC to the $\mathrm{CO}$ case, the specific energy sharing by user change substantially. With $\mathrm{CO}$, the assets installed by U2, U6 and U8 increase significantly and provide energy for most of the other users. Users U2 and U8 are also asked to install assets to supply more than their yearly demand, thanks to the cheap installation cost and source availability. Consumers U5, U7 and U10 instead withdraw most of the additional shared energy, followed by users U9, U1 and U4. Interestingly, these last users see their ratio of shared energy decrease between the ANC and CO case, as a greater share of renewable sources is available also by the other users, thus their specific contribution to the total shared energy decreases with respect to the ANC case. These results suggest that the configuration coordinated by the aggregator enables increasing self and shared consumption together with a larger production by renewable sources, thus inducing positive social attitudes while fostering environmental benefits.

\subsection{Investment costs and technical design}

The commitment in terms of required CAPEX and the total NPC of each user are specified in Fig. 6, aggregator included. It is worth noticing that the CAPEX and total NPC can change significantly among the different cases (NA, NC, ANC, or CO), especially for some users. In particular, in the CO sizing some 


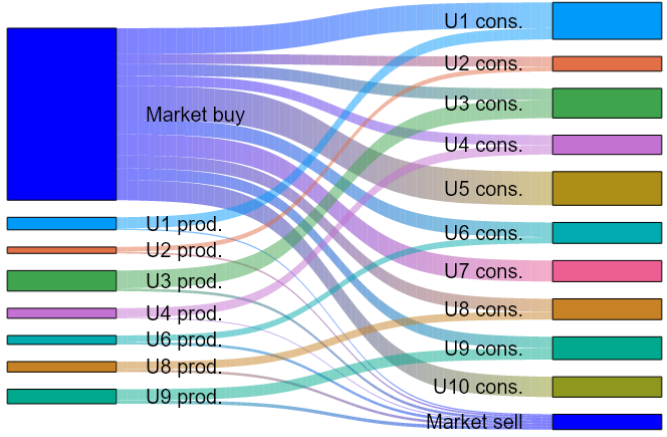

(a) Non-Cooperative (NC) case

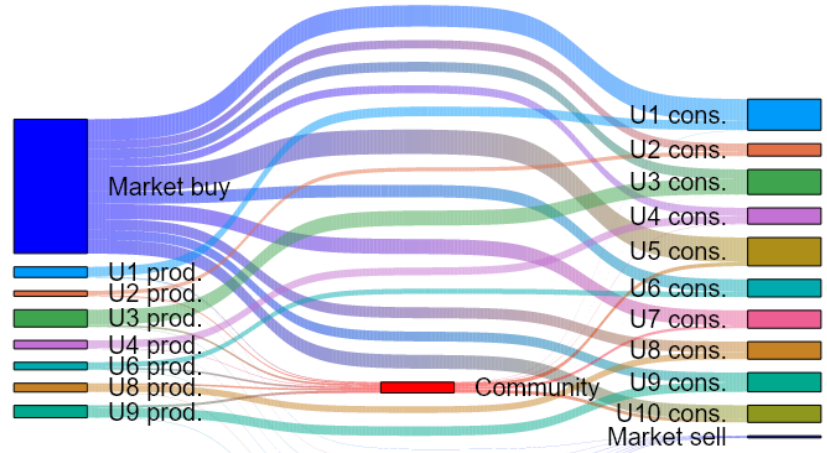

(b) Aggregated Non-Cooperative (ANC) case

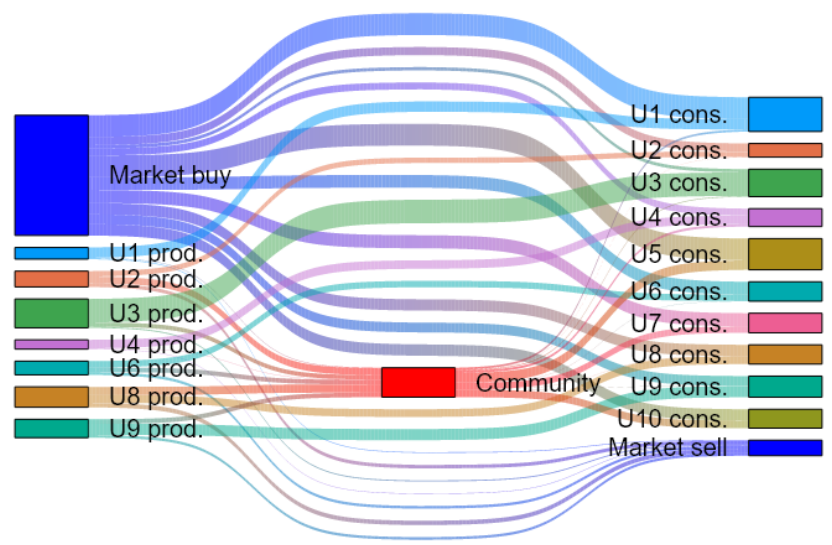

(c) Cooperative $(\mathrm{CO})$ case

Figure 4: Energy flows among users, the community and the public market.

users are suggested to heavily increase the investment in assets. For example, user U3 is asked to almost double its CAPEX investment to the benefit of significantly lower OPEX. The NPC differences between ANC and $\mathrm{CO}$ cases are instead related to the different reward distribution schemes, to be discussed in the following subsections.

The surge in CAPEX costs of the CO case (Fig. 3) is justified by a large increase in the installed assets with respect to NC/ANC cases, as shown in Fig. 7 and Fig. 8. User U3 is asked to more than double the size of his wind turbine, and similarly for the photovoltaic panels of U2. In the non-cooperative NC case, instead, installed assets are typically lower because the size of each asset is optimal for his owner user only, as there is no incentive in installing additional renewable assets or storage. Sometimes, the installed capacity of some users may also decrease because other sources may be cheaper, such as for user U3, whose PV CAPEX $(1600 € / \mathrm{kW})$ are higher than for other users U2, U4, U6, U8 or U9 $(1400 € / \mathrm{kW})$.

In order to maximize the self and shared consumption among the EC users, the installed capacity in 


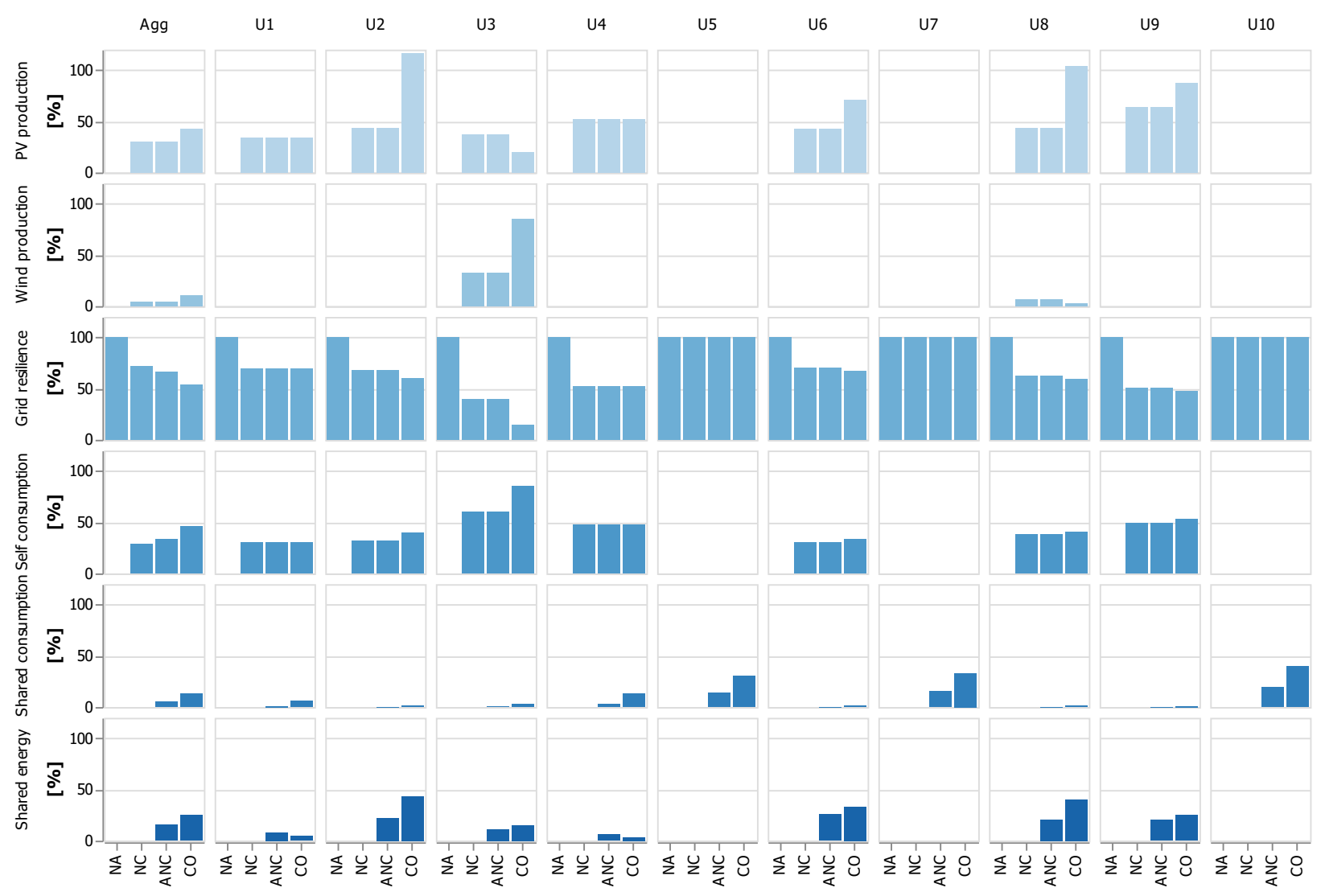

Figure 5: Energy (PV and wind) production, grid dependence, self/shared consumption and shared energy for the entire community (Agg) and each user (UX); values in percent of the yearly demand, except for "shared energy" that is normalized with respect to the yearly renewable production.

CAPEX NPC - CAPEX

Aggregator/User

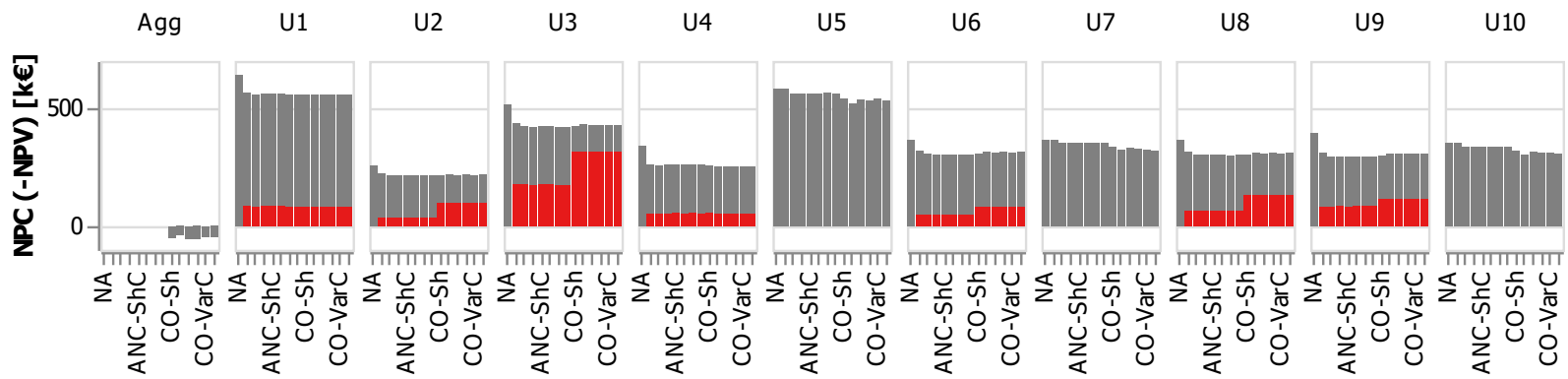

Figure 6: Net Present Cost and CAPEX by user and aggregator (negative values are revenues), for all configurations (NA, NC, ANC and CO) and reward distribution mechanisms (Sh, Nuc, ShC, ShNuc, VarC and VarNuc).

battery systems increases in the $\mathrm{CO}$ case with respect to the NC/ANC configurations, as shown in Fig. 8 . The general trend is that the added capacity reflects the installation of renewable assets, with the exception of user U6 due to higher CAPEX costs with respect to the other users. 


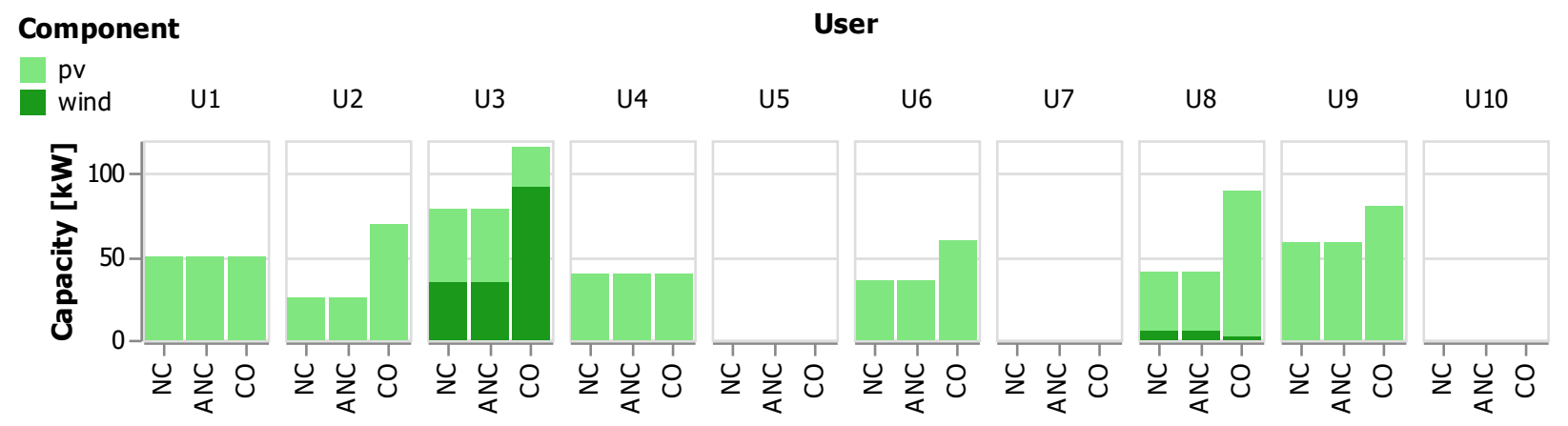

Figure 7: Renewable production by user.

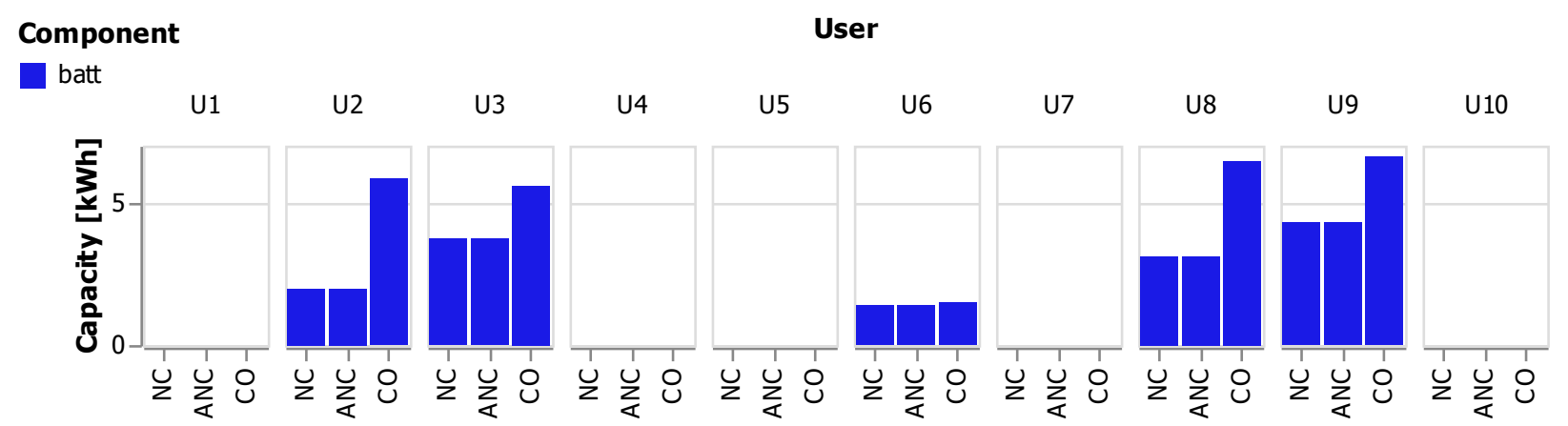

Figure 8: Battery capacity by user.

Therefore, these results further confirm the incentive-like effect that the EC framework is enabling, thus supporting both environmental and economic impacts. In particular, the proposed business model with the aggregator enables maximizing these targets, in agreement to the EU policies [10].

\subsection{Stability of the coalition}

The grand coalition is stable if the corresponding reward distribution belongs to the Core, or, equivalently, if the margins $\theta_{J}$ of all subcoalitions $J$ of the grand coalition are non-negative, as per their definition detailed in Section 4.1 and Section 4.3. Accordingly, we report in Table 2 the surplus $\underline{\theta}=\min \theta_{J}$ for the most "unhappy" sub-coalition (the one with the lowest surplus), and the total number of sub-coalitions whose $\theta_{J}$ is negative or null, according to the selected reward distribution. It is worth noticing that, in the CO configurations, the aggregator is always considered as a potential player in the EC, thus $J$ is subset of $I \cup\{A\}$. On the other hand, in the ANC case, we distinguish two cases: in columns 1-3, as per ANC definition, the aggregator is not a player in the market, thus $J \subset I$, while in columns 3-6, the same solution is then computed considering the aggregator as a player, i.e., $J$ is subset of $I \cup\{A\}$.

The results clearly show that in practice only the $\mathrm{CO}$ configuration can be considered stable (with all tested reward distributions, but the Shapley). Indeed, the ANC configurations reported with positive (minimal) margin are really stable only in the hypothesis that the aggregator irrationally and unilaterally decides to withdraw. At the very moment in which the aggregator announces its presence in the market, all configurations not including it become severely unstable. This suggests that any rational and well-informed user 
Table 2: Stability by configuration.

\begin{tabular}{c||ccc|ccc|cccccc}
\multicolumn{1}{c||}{} & \multicolumn{3}{c|}{ Without Agg } & \multicolumn{7}{c|}{ With Agg } & \multicolumn{7}{c}{ With Agg } \\
\hline Config. & ANC & ANC & ANC & ANC & ANC & ANC & CO & CO & CO & CO & CO & CO \\
Rew. dist. & Sh & Nuc & ShNuc & Sh & Nuc & ShNuc & Sh & Nuc & ShC & ShNuc & VarC VarNuc \\
\hline \hline $\min \theta_{J}[\mathrm{k} \in]$ & -3.0 & 0.3 & 0.3 & -117.4 & -121.8 & -121.3 & -12.6 & 2.3 & 0.0 & 2.3 & 0.0 & 2.3 \\
$\left|\left\{J: \theta_{J}<0\right\}\right|$ & 65 & 0 & 0 & 929 & 858 & 859 & 76 & 0 & 0 & 0 & 0 & 0 \\
$\left|\left\{J: \theta_{J}=0\right\}\right|$ & 0 & 0 & 0 & 2 & 2 & 2 & 0 & 0 & 7 & 0 & 6 & 0 \\
\hline Stable? & $\mathrm{N}$ & $\mathrm{Y}$ & $\mathrm{Y}$ & $\mathrm{N}$ & $\mathrm{N}$ & $\mathrm{N}$ & $\mathrm{N}$ & $\mathrm{Y}$ & $\mathrm{Y}$ & $\mathrm{Y}$ & $\mathrm{Y}$ & $\mathrm{Y}$
\end{tabular}

would rather join an EC well coordinated by an aggregator than join an ANC configuration, which goes in favour of the proposed methodology. Yet, in the following subsections the reward distribution of non-stable cases is still reported for comparison purposes.

It is worth observing that in the CO cases the Shapley value is never a stable configuration, contrarily to all the other tested mechanisms, which confirms that Shapley value may not belong to the Core. However, it can still be used as a reference value for profit revenue distribution, e.g. the Shapley/Core or Shapley/Nucleolus

cases. The other reward distribution mechanisms, instead, lead to stable configurations. However, it is worth noticing that, contrarily to the Core versions (ShC and $\operatorname{VarC}$ ), the Nucleolus-based formulations (Nuc, ShNuc and VarNuc) tend to provide a strictly positive margin, which means that even the most unhappy coalition can make $2.3 \mathrm{k} \in$ more profit within the grand coalition than without. Yet, this stronger stability may come at the partial cost of fairness. Instead, in the Core formulations, the fairness measure (Shapley or MinVariance criteria) is prioritized, within the limits of the Core; however, this leads to a small number (6-7) of sub-coalitions that are as profitable within the community than without, since they have a strictly null margin, which does not go in favour of the stability of the grand coalition. These results to suggest that a trade-off exists between fairness and stability of a coalition that needs to be properly managed to ensure the long-term viability of the EC.

\subsection{Fair reward allocation by user and aggregator}

In order to stress the analysis, in Fig. 9 we compare selected reward distribution mechanisms between the ANC and CO configurations, whereas in Fig. 10 we show the benefit allocation for all reward distributions (Shapley: Sh, Nucleolus: Nuc, Shapley/Core: ShC, MinVariance/Core: VarC, Shapley/Nucleolus: ShNuc and MinVariance/Nucleolus: VarNuc) for the CO configuration. Moreover, Table 3 details the specific benefit that may be fairly attributed to the aggregator on the same configurations.

First of all, it is worth noticing that in the CO configurations no less than $35 \mathrm{k} \in$ of benefits can be fairly attributed to the aggregator, as quantitatively shown in Table 3. This means that the aggregator is a strong enabler for cost reduction and thus its corresponding fair reward is relatively high. In fact, as shown in Fig. 3, costs reduce by about $100 \mathrm{k} €$ and $227 \mathrm{k} €$ with respect to the ANC and NC cases, respectively. However, the specific retribution of the aggregator slightly change depending on the reward distribution scheme. The Nucleolus approach is the least profitable for the aggregator, as it is about 15-33\% lower than any other tested reward distribution mechanism. The aggregator reward with ShC and ShNuc cases tend to be slighlty higher than the Shapley value, contrarily to VarC and VarNuc; however, differences are always within $10 \%$. 
This means that our methodology successfully quantifies the fair reward share for an aggregator of EC, under different criteria.

Given these results, we also quantified the parameter $\sigma$ of the model that allows, on one hand, to reduce the risks of the agency problem, as detailed in Section 3 and, on the other hand, to fairly reward the aggregator, as shown in Section 5. In particular, depending on the distribution scheme, the aggregator may retain between $16 \%$ and $24 \%$ the profits generated with respect to the $\mathrm{NC}$ case, or, equivalently, between $37 \%$ and $56 \%$ the benefits generated with respect to the ANC case. This suggests that this quantitative methodology can serve policy makers, developers and aggregators in tailoring policies and business options for the fair rewarding of aggregators in ECs.

Table 3: Fair benefit provided by the aggregator and calibration of $\sigma$ by reward distribution mechanism.

\begin{tabular}{|c|c|c|c|c|c|c|c|c|c|}
\hline $\begin{array}{l}\text { Config. } \\
\text { Rew. dist. }\end{array}$ & NA & $\mathrm{NC}$ & $\mathrm{ANC}$ & $\begin{array}{l}\mathrm{CO} \\
\mathrm{Sh}\end{array}$ & $\begin{array}{l}\mathrm{CO} \\
\mathrm{Nuc}\end{array}$ & $\begin{array}{l}\mathrm{CO} \\
\mathrm{ShC}\end{array}$ & $\begin{array}{c}\mathrm{CO} \\
\text { ShNuc }\end{array}$ & $\begin{array}{c}\mathrm{CO} \\
\operatorname{VarC}\end{array}$ & $\begin{array}{c}\mathrm{CO} \\
\text { VarNuc }\end{array}$ \\
\hline $\mathrm{SC}(-\mathrm{SW})[\mathrm{k} \in]$ & 4177.4 & 3734.9 & 3605.3 & 3507.8 & 3507.8 & 3507.8 & 3507.8 & 3507.8 & 3507.8 \\
\hline Cost diff. $[\mathrm{k} \Theta]$ & 442.4 & 0.0 (ref) & -129.6 & -227.1 & -227.1 & -227.1 & -227.1 & -227.1 & -227.1 \\
\hline Cost diff. [\%] & 11.8 & 0.0 (ref) & -3.5 & -6.1 & -6.1 & -6.1 & -6.1 & -6.1 & -6.1 \\
\hline $\operatorname{Agg}[\mathrm{k} \Theta]$ & - & - & - & 48.0 & 36.3 & 52.4 & 54.4 & 43.1 & 44.9 \\
\hline$\sigma[\%]$ & - & - & - & 21.1 & 16.0 & 23.1 & 24.0 & 19.0 & 19.8 \\
\hline
\end{tabular}

When looking in Fig. 9 at the specific benefit obtained by each user selected ANC and CO configurations with respect to the NC case, we noticed a pretty diversified situation. Moroever, as shown in Fig. 7 and Fig. 8, despite bearing most of the additional investment costs in renewable assets, users U2, U3, U6, U8 and U9 may be rewarded less with CO than with ANC, especially with the Nucleolus distribution scheme. This counterintuitive result can be well explained by (1) the intrinsic instabilities of ANC cases, well explained in Section 7.4 and (2) the instability of the Shapley Value, as discussed in Section 4. For example, users U5, U7 and U10 are worse off with the ANC cases than CO, thus they would prefer the latter; on the other hand, prosumers (e.g. U2, U3, U6, U8 and U9) cannot produce adequate reward without them absorbing their surplus energy. This further supports the idea that unstable coalitions rarely can emerge and the proposed stable CO configurations can properly support the formation of the grand coalitions.

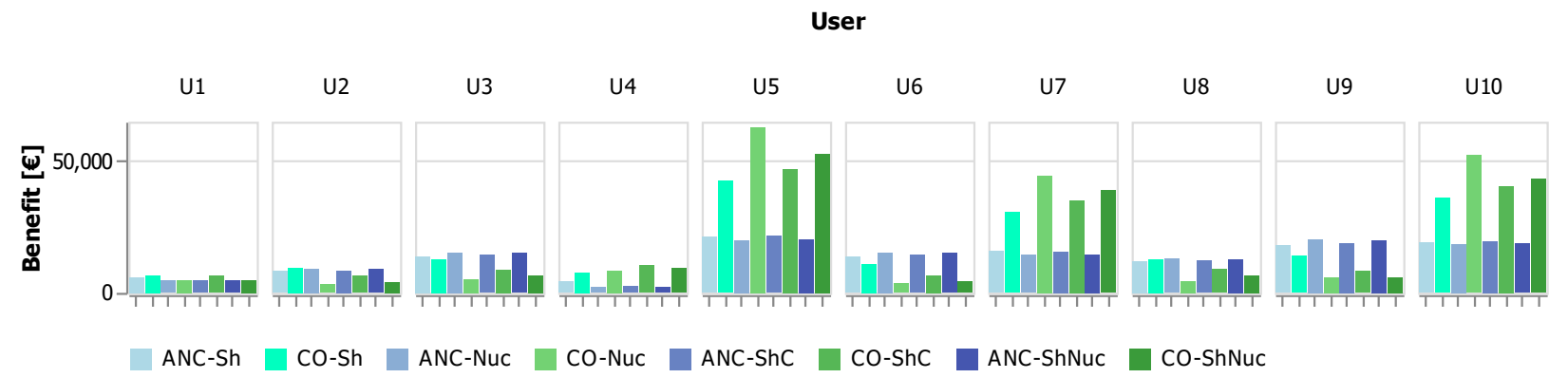

Figure 9: Benefit obtained by each user in $\mathrm{ANC}$ and $\mathrm{CO}$ configurations, with respect to the $\mathrm{NC}$ case, using different reward distribution mechanisms (Sh, Nuc, ShC and ShNuc).

Furthermore, Fig. 10 shows the reward distributions by user and aggregator for all stable CO configura- 
tions and the Shapley Value, which despite being unstable is still reported for the sake of comparison. All functions distribute profits with typical patters, but the Nucleolus-like (Nuc, ShNuc and VarNuc) methods tend to favour consumers over prosumers and the aggregator, conversely to Core-like (ShC and VarC) formulations and Shapley. In particular, the Nucleolus mechanism distributes profits to iteratively improve the margin for the least stable sub-coalition, and in this case, this means to largely improve the reward granted to consumers-only (U5, U7 and U10), which may be questionable; instead, in the ShNuc and VarNuc cases only the first most unhappy coalitions are considered. Furthermore, while Shapley-based methods (Sh, ShC and ShNuc) tend to align the reward distribution towards the result of the Shapley Value, the Variance method tends to spread rewards more uniformly, but users with higher NPC may be favoured, despite their contribution in the community, as shown for example in cases U7 and U10 where the reward distribution is higher than in the Shapley-based methods. Given the stability and fairness concerns, these results suggest that the modified Nucleolus approaches (ShNuc and VarNuc) propose both strictly stable distribution schemes and good fairness properties; when marginal stability is not a concern, also ShC and VarC methodologies can be considered, whereas the Nucleolus approach is very stable but may favuor consumers over prosumers, at least in our case study. The Shapley distribution mechanism, instead, is not stable, thus it may not support the formation of the grand coalition.

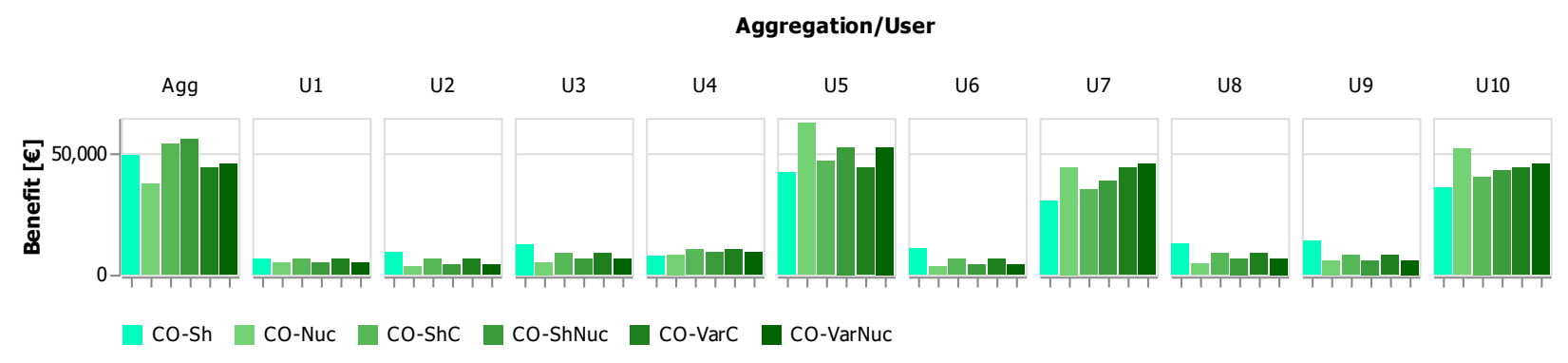

Figure 10: Benefit by user and aggregator for the $\mathrm{CO}$ configuration and reward distribution mechanisms.

\subsection{Exit clauses}

The exit costs, computed according to (54) and (55), are shown in Table 4 for the ANC and CO cases and representative reward allocation mechanisms. The surprising and unrealistic negative exit costs all correspond to the Shapley Value, which is never stable in the case study; this clearly-unintended behaviour, whereby users U2-U10 would be willing to pay user U1 up to $0.7 \mathrm{k} €$ to make him leave the aggregate, further strengthen the result that the Shapley rewarding scheme must not be used as-is. Indeed, this paradoxical issue does not occur in all other schemes, confirming their stability. However, it is worth noticing that, in the Nucleolus distribution, the maximum value of the exit clause is about $9 \mathrm{k} €$ and all users tend to have positive exit costs, whereas in the other cases the peak can be about $20-25 \mathrm{k} €$ and some users may have a null exit costs. This is because the Core/Shapley functions tend to privilege prosumers that support the reward creation more than consumers-only, conversely to Nucleolus, for the proposed case study. Therefore, the reward allocated to consumers-only tend to be lower than in the Nucleolus, but if that consumer leaves, the other users cannot collect the reward by supplying energy to him. Since in the Shapley-based cases a higher share of benefits is allocated to prosumers, when a consumer leaves the grand coalition the other prosumers would experience a larger economic loss with respect to Nucleolus-based methods, unless properly 
compensated by the exit costs. This justifies why in the Shapley-based methods the exit cost is generally larger than in the corresponding Nucleolus-based ones, especially for consumers. Since the EU rational for EC is related to increasing self/shared consumption and cooperation [27], our results indicate that the profit distribution schemes shall promote the formation of the grand coalition; then rational, stable and fair allocation mechanisms, such as the ShC, ShNuc, VarC and VarNuc or the Nucleolus functions, shall be recommended.

It is also worth noticing that leaving the EC during the first year is a very unlikely event; yet, even in this rare case, the exit costs can be acceptable as they are generally well below a typical user yearly bill, which is in the range $12-40 \mathrm{k} € / \mathrm{y}$ in the $\mathrm{NC}$ case and $19-50 \mathrm{k} € / \mathrm{y}$ in the NA configuration, depending on the user. However, as the table shows, exit costs are significantly reduced after 10 years, which is less than half of the expected life of most equipment of the EC. Moreover, the later a user leaves, the longer he benefits from the reduced costs enabled by the EC and the aggregator, which partially alleviate the burden of the exit costs. In particular, the cumulative avoided costs exceed the cost of leaving after about 10.1 years, therefore this number represents the break-even point after which a user can leave the community without being worse off with respect to the $\mathrm{NC}$ case.

Table 4: Economic value of the exit clause, by user and year of leaving.

\begin{tabular}{|c|c|c|c|c|c|c|c|c|c|c|c|}
\hline $\begin{array}{c}\text { Year } \\
{[y]}\end{array}$ & User & $\begin{array}{c}\mathrm{ANC} \\
\mathrm{Sh} \\
{[\mathrm{k} \in]}\end{array}$ & $\begin{array}{l}\mathrm{ANC} \\
\mathrm{Nuc} \\
{[\mathrm{k} \in]}\end{array}$ & $\begin{array}{l}\mathrm{ANC} \\
\mathrm{ShC} \\
{[\mathrm{k} \in]}\end{array}$ & $\begin{array}{c}\mathrm{ANC} \\
\text { ShNuc } \\
{[\mathrm{k} \in]}\end{array}$ & $\begin{array}{c}\mathrm{CO} \\
\mathrm{Sh} \\
{[\mathrm{k} \in]} \\
\end{array}$ & $\begin{array}{l}\mathrm{CO} \\
\mathrm{Nuc} \\
{[\mathrm{k} \in]}\end{array}$ & $\begin{array}{l}\mathrm{CO} \\
\mathrm{ShC} \\
{[\mathrm{k} \in]}\end{array}$ & $\begin{array}{c}\mathrm{CO} \\
\mathrm{ShNuc} \\
{[\mathrm{k} \in]}\end{array}$ & $\begin{array}{c}\mathrm{CO} \\
\operatorname{VarC} \\
{[\mathrm{k} \in]} \\
\end{array}$ & $\begin{array}{c}\mathrm{CO} \\
\operatorname{VarNuc} \\
{[\mathrm{k} \in]}\end{array}$ \\
\hline 1 & U1 & -0.7 & 0.4 & 0.4 & 0.5 & 0.3 & 2.3 & 0.7 & 2.3 & 0.4 & 2.3 \\
\hline 1 & U3 & 2.9 & 1.6 & 2.4 & 1.8 & -3.8 & 3.4 & 0.0 & 2.3 & 0.1 & 2.3 \\
\hline 1 & U5 & 5.0 & 6.2 & 4.7 & 5.9 & 29.2 & 9.2 & 24.8 & 19.0 & 27.7 & 19.0 \\
\hline 1 & U6 & 3.0 & 1.4 & 2.3 & 1.5 & -4.1 & 3.0 & 0.0 & 2.3 & 0.0 & 2.3 \\
\hline 1 & U10 & 4.5 & 5.0 & 3.9 & 4.8 & 24.1 & 8.3 & 19.7 & 16.8 & 16.3 & 14.5 \\
\hline 10 & U1 & -0.5 & 0.3 & 0.2 & 0.3 & 0.2 & 1.4 & 0.4 & 1.4 & 0.2 & 1.4 \\
\hline 10 & U3 & 1.8 & 1.0 & 1.5 & 1.1 & -2.4 & 2.1 & 0.0 & 1.4 & 0.1 & 1.4 \\
\hline 10 & U5 & 3.1 & 3.9 & 2.9 & 3.7 & 18.1 & 5.7 & 15.4 & 11.8 & 17.2 & 11.8 \\
\hline 10 & U6 & 1.8 & 0.9 & 1.4 & 0.9 & -2.6 & 1.9 & 0.0 & 1.4 & 0.0 & 1.4 \\
\hline 10 & U10 & 2.8 & 3.1 & 2.5 & 3.0 & 15.0 & 5.1 & 12.3 & 10.5 & 10.2 & 9.0 \\
\hline
\end{tabular}

\subsection{Effects on the peak power}

In Fig. 11, the effects of the different models on the peak power at both user and community level are reported. This is a specific benefit of our model, while the literature typically neglects the power effects and focuses on the energy sharing only [29]. In particular, on the left side of Fig. 11 we denote with "Agg" the average of the monthly peak power at MV/LV substation where the users are connected or, equivalently, at a virtual external POD all the users would be connected to. Instead, with "Tot peak" we refer to the average of the sum of the monthly peak energy flows at the user PODs, peaks that may occur at different times. It is clear that this latter value is always higher than the average monthly peak power ("Agg" peak) by construction, since the sum of the peaks of the user power profiles is higher than the peak of the sum of 
such power profiles. While comparing the different scenarios it is worth noticing that the higher the degree of integration of the users (from NA to $\mathrm{CO}$ ), the lower the net peak power at the substation: while the peak power at the $\mathrm{NC} / \mathrm{ANC}$ level is about $230 \mathrm{~kW}$, in the $\mathrm{CO}$ case this value decreases by about $8 \%(213 \mathrm{~kW})$. This suggests that although EC policies focus on energy sharing and do not directly focus on peak power measures, a total peak power usage at the distribution substation is achieved anyway, yet this is driven by the specific economic reward.

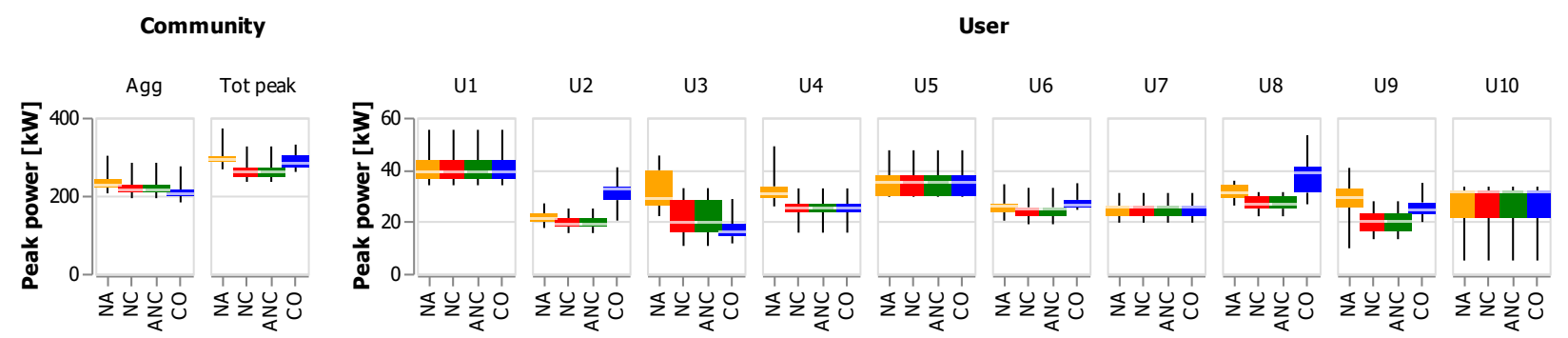

Figure 11: Average monthly peak power by user, aggregate (Agg) and the average of the sum of the monthly user peaks (Tot Peak); the white horizontal line represents the monthly average, the color bars represent the $25-\%$ and $75-\%$ percentiles, and the black bar depicts the maximum and minimum value.

Although the total net peak power decreases from the NC/ANC cases to CO, the total sum of the peak power by users (column "Tot peak") increases from $264 \mathrm{~kW}$ to $289 \mathrm{~kW}$, which means that, on average, each user increases the use of the local grid at its own POD, as confirmed by Fig. 11. In fact, the peak power of users $\mathrm{U} 2, \mathrm{U} 6, \mathrm{U} 8$ and $\mathrm{U} 9$ increases in the CO scenario with respect to the NC/ANC cases, and this contributes to enlarge the total sum of the peaks. However, as further suggested by Fig. 7, Fig. 8 and Fig. 4, this change is due to a higher renewable production, which meets a share of the demand of other users that would be withdrawn from the public grid, instead. Therefore, the net effect at the substation is a decrease in the total peak power w.r.t. the $\mathrm{ANC} / \mathrm{NC}$ cases, at least in the proposed case study. These results clearly show that the proposed business model can enable achieving higher coordination and self consumption that also lead to lower demand peaks at the distribution system, which is in favour of efficiency and deferred distribution investments.

\section{Conclusions}

This paper has successfully proposed both a novel business model and an optimization methodology for energy communities, accounting for fair revenue sharing policies and exit clauses. In particular we detailed the possible roles of aggregators in supporting ECs, a rewarding scheme to reduce the occurrence of the agency problem, a comparison with standard non-coordinated approaches without the aggregator, and a wide sensitivity analysis on game-theoretic reward allocation measures (Shapley Value, The Core, Nucleolus and a custom Shapley/Core approach), also evaluating the stability of the different mechanisms. A numerical case study for commercial applications has been developed for am Italian case study and supports the proposed business model and optimization strategy.

Compared to the NA scenario where no assets are installed, the cooperative $(\mathrm{CO})$ solution provided by the aggregator enables reducing total social costs by about $16 \%$. The cooperative solution is still $6 \%$ cheaper 
than the NC case where each user installs assets on his own. All this suggests that the aggregator supports value creation in ECs and for this reason it is recommended enabling aggregators to operate ECs, with a fair remuneration.

A sensitivity on the fair game-theoretic reward distribution schemes proves that Shapley value is not generally stable, conversely to Nucleolus function and to the proposed Shapley/Core, Shapley/Nucleolus, MinVariance/Core and Min/Variance Nucleolus. Results suggest that Nucleolus distribution scheme fosters stability by progressively maximizing the benefit of the most unhappy coalition, but tends to privilege passive members that do not install any assets. Conversely, Shapley Value, despite not being stable, distributes profits more evenly depending on each user contribution, thus privileging prosumers more than the Nucleolus. Instead, the proposed Shapley/Core and Shapley/Nucleolus successfully find a balance between these two positions, thus enabling strong stability and fairness. The MinVariance-based mechanisms behave similarly to the Shapley-based ones, yet the latter appeared to be slightly more fair.

Moreover, our results highlight that the fair remuneration of an aggregator can be about 16-23\% the savings generated with respect to the base case (NC). This value is regarded as a reasonable amount, also considering that users are awarded with the majority of the surplus, which is in the range $77-84 \%$, depending on the rewarding scheme. In particular, the Nucleolus is the least convenient for the aggregator, as it tends to favour consumers, whereas the proposed Shapley/Core is more rewarding for the aggregator. This result and the proposed methodology lay the foundation for fair rewarding schemes for aggregators of ECs and can support the activity of policy makers and business developers.

Furthermore, we also pointed out that the proposed business model and the aggregator can promote social, technical and environmental benefits, aligned to the EU policies: improved renewable penetration, from $35 \%$ ( $\mathrm{NC}$ case) up to $52.5 \%$ (CO case), and improved self-consumption, from $28 / 34 \%$ (NC/ANC cases) to $46 \%$ (CO case), among others. Interestingly, despite no direct economic benefit for the EC, we highlighted that the coordination promoted by an aggregator enables reducing also the peak power at the distribution substation (about $-8 \%$ with respect to the $\mathrm{NC} / \mathrm{ANC}$ cases).

These results confirm the positive effects enabled by ECs operated by aggregators, thus suggesting their important role and value for the development of EC policies and business applications. We also highlighted that the current EC framework can promote increased renewable penetration, but incentives may create distortions. In fact, while the coordinated solution leads to improved environmental, economic and social benefits, the users reap undeserved benefits also in non-coordinated solutions, such as in the ANC case. This study, revealing and quantifying this unintended effect, lays the foundation to research studies to tackle this issue.

All in all, we believe that our methodology can support policy makers and researchers in developing policy and technical recommendations for the development and tailoring of new measures and projects; furthermore, the proposed business model can also guide the development of commercial solutions.

\section{Acknowledgments}

Work carried out in the framework of the project "AUTENS" (Sustainable Energy Autarky), funded by the University of Pisa (PRA 2020 program). 


\section{References}

[1] Ibrahim Abada, Andreas Ehrenmann, and Xavier Lambin. On the viability of energy communities. Energy Journal, 2020.

[2] Ibrahim Abada, Andreas Ehrenmann, and Xavier Lambin. Unintended consequences: The snowball effect of energy communities. Energy Policy, 143(March):111597, 2020.

[3] Uzma Amin, Jahangir Hossain, Wayes Tushar, and Khizir Mahmud. Energy Trading in Local Electricity Markets with Renewables- A Contract Theoretic Approach. IEEE Transactions on Industrial Informatics, 17(6):1-1, 2020.

[4] ARERA. Obiettivi Strategici e linee di intervento 2019-2021: Area Energia.

[5] Edward Barbour, David Parra, Zeyad Awwad, and Marta C. González. Community energy storage: A smart choice for the smart grid? Applied Energy, 2018.

[6] Gerald W. Braun. State policies for collaborative local renewable integration. Electricity Journal, 33(1):106691, 2020.

[7] Vasco Brummer. Community energy - benefits and barriers: A comparative literature review of Community Energy in the UK, Germany and the USA, the benefits it provides for society and the barriers it faces. Renewable and Sustainable Energy Reviews, 94(June):187-196, 2018.

[8] Scott Burger, Jose Pablo Chaves-Ávila, Carlos Batlle, and Ignacio J Pérez-Arriaga. A review of the value of aggregators in electricity systems. Renewable and Sustainable Energy Reviews, 77:395-405, 2017.

[9] Pedro Calleja and Francesc Llerena. Consistency, weak fairness, and the Shapley value. Mathematical Social Sciences, 105:28-33, 2020.

[10] Aura Caramizaru and Andreas Uihlein. Energy communities : an overview of energy and social innovation. Technical report, JRC, 2019.

[11] Pratyush Chakraborty, Enrique Baeyens, Kameshwar Poolla, Pramod P. Khargonekar, and Pravin Varaiya. Sharing Storage in a Smart Grid: A Coalitional Game Approach. IEEE Transactions on Smart Grid, 10(4):4379-4390, 2019.

[12] Georgios Chalkiadakis, Edith Elkind, and Michael Wooldridge. Cooperative game theory: Basic concepts and computational challenges. IEEE Intelligent Systems, 27(3):86-90, 2012.

[13] Bo Chen, Jianhui Wang, Xiaonan Lu, Chen Chen, and Shijia Zhao. Networked Microgrids for Grid Resilience, Robustness, and Efficiency: A Review. IEEE Transactions on Smart Grid, 12(1):18-32, 2021.

[14] Adriana Chis and Visa Koivunen. Coalitional game-based cost optimization of energy portfolio in smart grid communities. IEEE Transactions on Smart Grid, 2019.

[15] Bertrand Cornélusse, Iacopo Savelli, Simone Paoletti, Antonio Giannitrapani, and Antonio Vicino. A community microgrid architecture with an internal local market. Applied Energy, 242(April):547-560, 2019 . 
[16] Pablo Cortés, Paloma Auladell-León, Jesús Muñuzuri, and Luis Onieva. Near-optimal operation of the distributed energy resources in a smart microgrid district. Journal of Cleaner Production, 252:119772, 2020 .

[17] Saeed Rahmani Dabbagh and Mohammad Kazem Sheikh-El-Eslami. Risk-based profit allocation to DERs integrated with a virtual power plant using cooperative Game theory. Electric Power Systems Research, 121:368-378, 2015.

[18] Yan Du, Zhiwei Wang, Guangyi Liu, Xi Chen, Haoyu Yuan, Yanli Wei, and Fangxing Li. A cooperative game approach for coordinating multi-microgrid operation within distribution systems. Applied Energy, 222(December 2017):383-395, 2018.

[19] R. Giglioli and D. Poli. Small-scale biomass-fired cogeneration, pellet production or district heating: New criteria for selecting the most profitable solution. International Review on Modelling and Simulations, 6(3), 2013.

[20] M. Giuntoli and D. Poli. Optimized Thermal and Electrical Scheduling of a Large Scale Virtual Power Plant in the Presence of Energy Storages. IEEE Trans. Smart Grid, 4(2):942 - 955, 2013.

[21] Vladimir Z. Gjorgievski, Snezana Cundeva, and George E. Georghiou. Social arrangements, technical designs and impacts of energy communities: A review. Renewable Energy, 169:1138-1156, 2021.

[22] Liyang Han, Thomas Morstyn, and Malcolm McCulloch. Incentivizing Prosumer Coalitions With Energy Management Using Cooperative Game Theory. IEEE Transactions on Power Systems, 34(1):303-313, 2019.

[23] Christina E. Hoicka and Julie L. MacArthur. From tip to toes: Mapping community energy models in Canada and New Zealand. Energy Policy, 121(June):162-174, 2018.

[24] Binod Prasad Koirala, Elta Koliou, Jonas Friege, Rudi A. Hakvoort, and Paulien M. Herder. Energetic communities for community energy: A review of key issues and trends shaping integrated community energy systems. Renewable and Sustainable Energy Reviews, 56:722-744, 2016.

[25] Despina Koraki and Kai Strunz. Wind and solar power integration in electricity markets and distribution networks through service-centric virtual power plants. IEEE Transactions on Power Systems, 33(1):473$485,2018$.

[26] Junkai Liang, Ashwin Shirsat, and Wenyuan Tang. Sustainable community based PV-storage planning using the Nash bargaining solution. International Journal of Electrical Power and Energy Systems, 118(November 2019):105759, 2020.

[27] J. Lowitzsch, C. E. Hoicka, and F. J. van Tulder. Renewable energy communities under the 2019 European Clean Energy Package - Governance model for the energy clusters of the future? Renewable and Sustainable Energy Reviews, 122:109489, apr 2020.

[28] Tomasz P. Michalak, Karthik V. Aadithya, Piotr L. Szczepanski, Balaraman Ravindran, and Nicholas R. Jennings. Efficient computation of the shapley value for game-theoretic network centrality. Journal of Artificial Intelligence Research, 46:607-650, 2013. 
[29] Matteo Moncecchi, Stefano Meneghello, and Marco Merlo. A game theoretic approach for energy sharing in the italian renewable energy communities. Applied Sciences (Switzerland), 10(22):1-25, 2020.

[30] Mahdi Motalleb and Reza Ghorbani. Non-cooperative game-theoretic model of demand response aggregator competition for selling stored energy in storage devices. Applied Energy, 202:581-596, 2017.

[31] Noemí Navarro and Andres Perea. A simple bargaining procedure for the myerson value. B.E. Journal of Theoretical Economics, 2013.

[32] H T Nguyen and L B Le. Sharing Profit from Joint Offering of a Group of Wind Power Producers in Day Ahead Markets. IEEE Transactions on Sustainable Energy, 9(4):1921-1934, 2018.

[33] Hung Khanh Nguyen, Hamed Mohsenian-Rad, Amin Khodaei, and Zhu Han. Decentralized Reactive Power Compensation Using Nash Bargaining Solution. IEEE Transactions on Smart Grid, 8(4):1679$1688,2017$.

[34] C. Nolden, J. Barnes, and J. Nicholls. Community energy business model evolution: A review of solar photovoltaic developments in England. Renewable and Sustainable Energy Reviews, 122(May 2019):109722, 2020.

[35] Gearóid O'Brien, Abbas El Gamal, and Ram Rajagopal. Shapley Value Estimation for Compensation of Participants in Demand Response Programs. IEEE Transactions on Smart Grid, 6(6):2837-2844, 2015.

[36] Guillermo Owen. On the core of linear production games. Mathematical Programming, 9(1):358-370, 1975.

[37] A. T.D. Perera, Z. Wang, Vahid M. Nik, and Jean Louis Scartezzini. Towards realization of an Energy Internet: Designing distributed energy systems using game-theoretic approach. Applied Energy, 283(November 2020):116349, 2021.

[38] Stefan Pfenninger and Iain Staffell. Long-term patterns of European PV output using 30 years of validated hourly reanalysis and satellite data. Energy, 114:1251-1265, 2016.

[39] Kris Poncelet, Hanspeter Hoschle, Erik Delarue, Ana Virag, and William Drhaeseleer. Selecting representative days for capturing the implications of integrating intermittent renewables in generation expansion planning problems. IEEE Transactions on Power Systems, 32(3):1936-1948, 2017.

[40] Matthew Rabin. Incorporating fairness into game theory and economics. In Advances in Behavioral Economics. 2011.

[41] M Rayati, M Bozorg, and R Cherkaoui. Coordinating strategic aggregators in an active distribution network for providing operational flexibility. Electric Power Systems Research, 189, 2020.

[42] David Schmeidler. The Nucleolus of a Characteristic Function Game. SIAM J. on Applied Mathematics, 1969 .

[43] Lloyd S. Shapley. The value of an n-Person Game, 1953.

[44] Chester S. Spatt. Conflicts of Interest in Asset Management and Advising, 2020. 
[45] Jan Stede, Karin Arnold, Christa Dufter, Georg Holtz, Serafin von Roon, and Jörn C Richstein. The role of aggregators in facilitating industrial demand response: Evidence from Germany. Energy Policy, 147:111893, 2020.

[46] Jeongmeen Suh and Sung-Guk Yoon. Profit-Sharing Rule for Networked Microgrids Based on Myerson Value in Cooperative Game. IEEE Access, 9:5585-5597, 2020.

[47] M A Tajeddini, H Kebriaei, and L Glielmo. Decentralized Hierarchical Planning of PEVs Based on Mean-Field Reverse Stackelberg Game. IEEE Transactions on Automation Science and Engineering, 17(4):2014-2024, 2020.

[48] Mao Tan, Yuqing Zhou, Ling Wang, Yongxin Su, Bin Duan, and Rui Wang. Fair-efficient energy trading for microgrid cluster in an active distribution network. Sustainable Energy, Grids and Networks, $26: 100453,2021$.

[49] Artur Trindade. Electricity Load Diagrams, 2015.

[50] Niklas Vespermann, Thomas Hamacher, and Jalal Kazempour. Access Economy for Storage in Energy Communities. IEEE Transactions on Power Systems, 8950(c):1-1, 2020.

[51] Hao Wang and Jianwei Huang. Cooperative Planning of Renewable Generations for Interconnected Microgrids. IEEE Transactions on Smart Grid, 7(5):2486-2496, 2016.

[52] Lu Wang, Wei Gu, Zhi Wu, Haifeng Qiu, and Guangsheng Pan. Non-cooperative game-based multilateral contract transactions in power-heating integrated systems. Applied Energy, 268(October 2019):114930, 2020 .

[53] Yahui Wang, Xiaorui Wu, Yong Li, Ruifeng Yan, Yi Tan, Xuebo Qiao, and Yijia Cao. Autonomous energy community based on energy contract. IET Generation, Transmission and Distribution, 14(4):682-689, 2020 .

[54] Juan M. Zolezzi and Hugh Rudnick. Transmission cost allocation by cooperative games and coalition formation. IEEE Transactions on Power Systems, 17(4):1008-1015, 2002. 